\title{
Stability analysis for the virtual element method
}

\author{
Lourenço Beirão da Veiga \\ Dipartimento di Matematica e Applicazioni, \\ Università di Milano-Bicocca, Via Cozzi 53, \\ I-20125, Milano, Italy \\ IMATI del CNR, Via Ferrata 1, 27100 Pavia, Italy \\ lourenco.beirao@unimib.it \\ Carlo Lovadina* \\ Dipartimento di Matematica, Università degli Studi di Milano, \\ Via Saldini 50, I-20133, Milano, Italy \\ IMATI del CNR, Via Ferrata 1, 27100 Pavia, Italy \\ carlo.lovadina@unimi.it \\ Alessandro Russo \\ Dipartimento di Matematica e Applicazioni, \\ Università di Milano-Bicocca, Via Cozzi 53, \\ I-20125, Milano, Italy \\ IMATI del CNR, Via Ferrata 1, 27100 Pavia, Italy \\ alessandro.russo@unimib.it
}

Received 19 July 2016

Revised 29 May 2017

Accepted 7 August 2017

Published 21 September 2017

Communicated by F. Brezzi

\begin{abstract}
We analyze the virtual element methods (VEM) on a simple elliptic model problem, allowing for more general meshes than the one typically considered in the VEM literature. For instance, meshes with arbitrarily small edges (with respect to the parent element diameter) can be dealt with. Our general approach applies to different choices of the stability form, including, for example, the "classical" one introduced in Ref. 4, and a recent one presented in Ref. 34 Finally, we show that the stabilization term can be simplified by dropping the contribution of the internal-to-the-element degrees of freedom. The resulting stabilization form, involving only the boundary degrees of freedom, can
\end{abstract}

* Corresponding author

This is an open access article published by World Scientific Publishing and distributed under the terms of the Creative Commons Attribution (CC BY) 4.0 License, which permits use, distribution and reproduction in any medium, provided the original author(s) and source are credited. 
be used in the VEM scheme without affecting the stability and convergence properties. The numerical tests are in accordance with the theoretical predictions.

Keywords: Virtual element methods; stability analysis; convergence analysis.

AMS Subject Classification: 65N12, 65N30, 65N15

\section{Introduction}

The virtual element method (VEM) has been introduced recently in Refs. 4, 5, [16 and 1] as a generalization of the finite element method that allows to make use of general polygonal/polyhedral meshes. The VEM, that enjoyed an increasing interest in the recent literature, has been developed in many aspects and applied to many different problems; we here cite only a few works (Refs. 8, 6, 17, 11, 12, 15, 24. 17 27 28, 10, 34 and [33) in addition to the ones above, without pretending to be exhaustive. We also note that VEM is not the only recent method that can make use of polytopal meshes: we refer, again as a minimal sample list of papers, to Refs. 18, 19, 20, 23, 29, 31 and 32 .

A VEM scheme may be seen as a Galerkin method built by means of two parts:

(1) a first term strongly consistent on polynomials, which guarantees the accuracy;

(2) a stabilization term $s_{E}(\cdot, \cdot)$, involving a suitably designed bilinear form.

We remark that under the usual assumptions on the polygonal mesh (namely, shape regularity and the property that in a polygon, the length of each edge is uniformly comparable to the diameter), devising and proving the stability features of the form $s_{E}(\cdot, \cdot)$ is quite simple. This is the reason why, in the VEM literature, the focus is on describing explicit expression for $s_{E}(\cdot, \cdot)$, while the proof of the corresponding stability result is often omitted. Instead, the stability analysis is more involved if one allows for more general mesh assumptions (for instance dropping the edge length condition mentioned above).

This paper focuses on the stability properties of the bilinear form $s_{E}(\cdot, \cdot)$. Although the approach we follow is quite general, we here consider the problem and notation of Refs. 4 and 6 in order to keep the presentation clearer. Our main results are the following.

- The development of a new strategy to prove the convergence of the VEM schemes, which requires weaker stability conditions on $s_{E}(\cdot, \cdot)$ than the usual ones. Our approach is used to analyze the situations described below:

(1) VEM schemes using a sequence of meshes with minor restrictions than the ones usually requested. In particular, our analysis covers some instance of shape regular meshes with edges arbitrarily short with respect to the diameters of the elements they belong to.

(2) Different instances of stabilization forms $s_{E}(\cdot, \cdot)$. Among them, we provide a detailed analysis of both the classical choice presented in Refs. 4] and 6] and a new one proposed in Ref. 34. In addition, it is worth remarking that a 
stability analysis for this latter choice could be developed using the tools of Ref. 4. However, the resulting error bound would be sub-optimal, in contrast with the numerical evidences. Our new approach, instead, leads to establish error bounds in perfect accordance with the numerical tests. We also show that the choice presented in Ref. [34, can have superior robustness properties in the presence of small edges.

- The development of a stability result concerning the choice of $s_{E}(\cdot, \cdot)$ presented in Refs. 4 and 6] that is valid under more general mesh assumptions. Essentially, we prove that the stabilization term is equivalent to the $H^{1}$-seminorm, where one of the two equivalence constants logarithmically degenerates in presence of "small" edges.

- An interesting result regarding the structure of $s_{E}(\cdot, \cdot)$. More precisely, we prove that the internal term $s_{E}^{\circ}(\cdot, \cdot)$ can be dropped without any detriment to the stability features of the underlying VEM scheme.

A brief outline of the paper is as follows. In Sec. 2, we present the continuous model problem, we review its virtual element discretization and we summarize the main results of the paper. In Sec. 3, we present and develop our error analysis strategy. Afterwards, in Sec. 4 we apply such an approach in order to analyze some existing choices of the stability form, under more general mesh assumptions than the ones typically adopted in the VEM literature. We present some numerical tests in Sec. 5. Finally, a set of basic, yet important, technical tools are detailed in Sec. 6]

\section{The Continuous and Discrete Problems}

In this section, we briefly present the continuous problem and its discretization with the VEM. More details can be found in Refs. 4 and 6 .

\subsection{The continuous problem}

As a model elliptic problem we consider the diffusion problem in primal form. Defining $(\cdot, \cdot)$ as the scalar product in $L^{2}$, and $a(u, v):=(K \nabla u, \nabla v)$, the variational formulation of the problem reads:

$$
\left\{\begin{array}{l}
\text { Find } u \in V:=H_{0}^{1}(\Omega) \quad \text { such that } \\
a(u, v)=(f, v) \quad \forall v \in V,
\end{array}\right.
$$

where $\Omega \subset \mathbb{R}^{2}$ is a polygonal domain and the loading $f \in L^{2}(\Omega)$. The diffusion symmetric tensor $K=K(x, y)$ is assumed to satisfy:

$$
c|\boldsymbol{\xi}|^{2} \leq \boldsymbol{\xi} \cdot K(x, y) \boldsymbol{\xi} \leq C|\boldsymbol{\xi}|^{2}, \quad \forall \boldsymbol{\xi} \in \mathbb{R}^{2}, \quad \forall(x, y) \in \Omega
$$

Above, $|\cdot|$ denotes the Euclidean norm in $\mathbb{R}^{2}$, while $c$ and $C$ are positive constants. 
It is well known that problem (2.1) has a unique solution, because our assumptions on $K$ and the Poincaré inequality yield:

$$
a(u, v) \leq M\|u\|_{H^{1}(\Omega)}\|v\|_{H^{1}(\Omega)}, \quad a(v, v) \geq \alpha\|v\|_{H^{1}(\Omega)}^{2} \quad \forall u, v \in V,
$$

with $0<\alpha \leq M<+\infty$.

Note that the bilinear form $a(\cdot, \cdot)$ in $(2.2)$ can obviously be split as:

$$
a(v, w)=\sum_{E \in \mathcal{T}_{h}} a_{E}(v, w) \quad \text { with } \quad a_{E}(v, w):=\int_{E} K \nabla v \cdot \nabla w
$$

for all $v, w \in V$.

\subsection{The virtual element method}

Let $k$ be an integer, equal or greater than 1, and let $\left\{\Omega_{h}\right\}_{h}$ denote a family of meshes, made of general simple polygons, on $\Omega$. Given an element $E \in \Omega_{h}$ of diameter $h_{E}$ and area $|E|$, its boundary $\partial E$ is subdivided into $N=N(E)$ straight segments, which are called edges, with a little abuse of terminology. Accordingly, the endpoints of the edges are called vertices of the element $E$. We remark that several consecutive edges of $E$ may be collinear; as a consequence, the number of edges (and vertices) may be greater than the minimum number of straight segments covering $\partial E$. Hence, a triangle may have 10 edges, for instance. Furthermore, the length of an edge $e \in \partial E$ is denoted by $h_{e}$. Moreover, in the sequel we assume that the diffusion tensor $K$ is piecewise constant with respect to the meshes $\left\{\Omega_{h}\right\}_{h}$.

For each $E \in \Omega_{h}$, we now introduce the local virtual space

$$
V_{E}=\left\{v_{h} \in H^{1}(E) \cap C^{0}(E):-\Delta v_{h} \in \mathbb{P}_{k-2}(E), v_{h \mid e} \in \mathbb{P}_{k}(e) \forall e \in \partial E\right\},
$$

where $\mathbb{P}_{n}, n \in \mathbb{N}$, denotes the polynomial space of degree $n$, with the convention that $\mathbb{P}_{-1}=\{0\}$. The associated set of local degrees of freedom $\Xi$ (divided into boundary ones $\Xi^{\partial}$, and internal ones $\Xi^{\circ}$ ) are given by:

- point values at the vertices of $E$;

- for each edge, point values at $(k-1)$ distinct points on the edge (this are typically taken as the internal Gauss-Lobatto nodes, see Refs. 4 and 6);

- the internal moments against a scaled polynomial basis $\left\{m_{i}\right\}_{i=1}^{k(k-1) / 2}$ of $\mathbb{P}_{k-2}(E)$ :

$$
\Xi_{i}^{\circ}\left(v_{h}\right)=|E|^{-1} \int_{E} v_{h} m_{i}, \quad \operatorname{span}\left\{m_{i}\right\}_{i=1}^{k(k-1) / 2}=\mathbb{P}_{k-2}(E), \quad\left\|m_{i}\right\|_{L^{\infty}(E)} \simeq 1 .
$$

For future reference, we collect all the $N k$ boundary degrees of freedom (the first two items above) and denote them with $\left\{\Xi_{i}^{\partial}\right\}_{i=1}^{N k}$.

The global space $V_{h} \subseteq H_{0}^{1}(\Omega)$ (such that $V_{h \mid E}=V_{E}$ ) is obtained by gluing the above spaces, and the same holds for the global degrees of freedom. We refer to Ref. 4 for the explicit expression. On each element $E$ we also define a projector 
$\Pi_{E}^{\nabla}: V_{E} \rightarrow \mathbb{P}_{k}(E)$, orthogonal with respect to the bilinear form $a_{E}(\cdot, \cdot)$. More explicitly, for all $v_{h} \in V_{E}$ :

$$
\left\{\begin{array}{l}
\Pi_{E}^{\nabla} v_{h} \in \mathbb{P}_{k}(E), \\
a_{E}\left(v_{h}-\Pi_{E}^{\nabla} v_{h}, p\right)=0 \quad \forall p \in \mathbb{P}_{k}(E), \\
\mathcal{R}\left(v_{h}-\Pi_{E}^{\nabla} v_{h}\right)=0,
\end{array}\right.
$$

where $\mathcal{R}$ denotes any projection operator onto the space $\mathbb{P}_{0}(E)$. In the literature, one can find various choices for the operator $\mathcal{R}$. We here focus on a popular choice, that makes use of an average on the boundary (for other possible choices see Remark 2.2):

$$
\mathcal{R} v_{h}=|\partial E|^{-1} \int_{\partial E} v_{h}
$$

It is easy to check that the above projector $\Pi_{E}^{\nabla}$ is computable on the basis of the available degrees of freedom (see Ref. 4). Moreover, we introduce the following symmetric and positive semi-definite stability bilinear form on $V_{E} \times V_{E}$ :

$$
s_{E}\left(v_{h}, w_{h}\right)=s_{E}^{\partial}\left(v_{h}, w_{h}\right)+s_{E}^{\circ}\left(v_{h}, w_{h}\right) .
$$

Equation (2.7) highlights that $s_{E}$ is the sum of two contributions: the first, $s_{E}^{\partial}$, involving the boundary degrees of freedom; the second, $s_{e}^{\circ}$, involving the internal degrees of freedom. For $s_{e}^{\circ}$, the classical choice is:

$$
s_{E}^{\circ}\left(v_{h}, w_{h}\right)=\sum_{i=1}^{k(k-1) / 2} \Xi_{i}^{\circ}\left(v_{h}\right) \Xi_{i}^{\circ}\left(w_{h}\right) .
$$

As far as $s_{E}^{\partial}$ is concerned, we consider the following two options:

$$
\begin{aligned}
& s_{E}^{\partial}\left(v_{h}, w_{h}\right)=\sum_{i=1}^{N k} \Xi_{i}^{\partial}\left(v_{h}\right) \Xi_{i}^{\partial}\left(w_{h}\right) \quad \text { (classical choice); } \\
& s_{E}^{\partial}\left(v_{h}, w_{h}\right)=h_{E} \int_{\partial E} \partial_{s} v_{h} \partial_{s} w_{h} d s \quad \text { (choice proposed in Ref. 34). }
\end{aligned}
$$

Now we can define the local discrete bilinear forms on $V_{E} \times V_{E}$ :

$$
a_{E}^{h}\left(v_{h}, w_{h}\right)=a_{E}\left(\Pi_{E}^{\nabla} v_{h}, \Pi_{E}^{\nabla} w_{h}\right)+s_{E}\left(\left(I-\Pi_{E}^{\nabla}\right) v_{h},\left(I-\Pi_{E}^{\nabla}\right) w_{h}\right)
$$

that are computable and approximate $a_{E}(\cdot, \cdot)$. Given the global discrete form

$$
a^{h}\left(v_{h}, w_{h}\right)=\sum_{E \in \Omega_{h}} a_{E}^{h}\left(v_{h \mid E}, w_{h \mid E}\right) \quad \forall v_{h}, w_{h} \in V_{h},
$$

the discrete problem is:

$$
\left\{\begin{array}{l}
\text { Find } u_{h} \in V_{h}, \\
a^{h}\left(u_{h}, v_{h}\right)=\left\langle f_{h}, v_{h}\right\rangle \quad \forall v_{h} \in V_{h} .
\end{array}\right.
$$

For a discussion about the approximated loading term $\left\langle f_{h}, v_{h}\right\rangle$, we refer to Refs. 4 and 1. In order to shorten the notation, and also to underline the generality 
of the proposed approach, in the following we will simply use $\Pi_{E}$ instead of $\Pi_{E}^{\nabla}$ to denote the projector operator.

Remark 2.1. The stability form $s_{E}(\cdot, \cdot)$ may also be scaled by a multiplicative factor $\tau_{E}>0$, to take into account the magnitude of the material parameter $K$, for instance. In this respect, a possible choice could be to set $\tau_{E}$ as the trace of $K$ on each element. In this paper, we do not deeply investigate on how to select $\tau_{E}$, but we address the reader to Refs. [5, 24 and 8 for some study on such an issue.

Remark 2.2. In addition to (2.6), a pair of other popular choices for the operator $\mathcal{R}$ are the following. A typical one, that can be used for $k \geq 2$, is given by the average on the element $E$ :

$$
\mathcal{R} v_{h}=|E|^{-1} \int_{E} v_{h}
$$

Another choice, valid for any $k$, is the average of the vertex values

$$
\mathcal{R} v_{h}=\frac{1}{N} \sum_{i=1}^{N} v_{h}\left(p_{i}\right),
$$

where the $p_{i}$ 's denote the vertices of $E$. The analysis of this paper easily extends, with minor modifications, also to the two cases here above. The details of the proofs can be found in Ref. 9.

\subsection{Mesh assumptions and overview of the main results}

In this section, we present the mesh assumptions considered in the paper, along with a table summarizing the main results.

We will deal with the following three mesh assumptions.

(A1) It exists $\gamma \in \mathbb{R}^{+}$such that all elements $E$ of the mesh family $\left\{\Omega_{h}\right\}_{h}$ are star-shaped with respect to a ball $B_{E}$ of radius $\rho_{E} \geq \gamma h_{E}$ and center $\mathbf{x}_{E}$.

(A2) It exists $C \in \mathbb{N}$ such that $N(E) \leq C$ for all elements $E \in\left\{\Omega_{h}\right\}_{h}$.

(A3) It exists $\eta \in \mathbb{R}^{+}$such that for all elements $E$ of the mesh family $\left\{\Omega_{h}\right\}_{h}$ and all edges $e \in \partial E$ it holds $h_{e} \geq \eta h_{E}$.

In all the presented results we will explicitly write which of those hypotheses are used, if any.

We remark that assumptions (A1) and (A3) are those considered in Ref. 4 Here, we want to consider also weaker assumptions in terms of the edge requirements, namely the combination of (A1) and (A2). It is easy to check that, provided (A1) holds, assumption (A3) implies (A2). However, assumption (A2) is much weaker than (A3), as it allows for edges arbitrarily small with respect to the element diameter.

In the following the symbol $\lesssim$ will denote a bound up to a constant that is uniform for all $E \in\left\{\Omega_{h}\right\}_{h}$ (but may depend on the polynomial degree $k$ ). Moreover, 
Table 1. Scheme of the convergence results. The row determines the mesh assumptions, while the column the type of stabilization boundary term. $h_{m(E)}$ denotes the length of the smallest edge of $E$.

\begin{tabular}{|c|c|c|}
\hline & $\begin{array}{l}s_{E}^{\partial}\left(v_{h}, w_{h}\right)= \\
\quad \sum_{i=1}^{N k} \Xi_{i}^{\partial}\left(v_{h}\right) \Xi_{i}^{\partial}\left(w_{h}\right)\end{array}$ & $\begin{array}{l}s_{E}^{\partial}\left(v_{h}, w_{h}\right)= \\
\quad h_{E} \int_{\partial E} \partial_{s} v_{h} \partial_{s} w_{h} \mathrm{~d} s\end{array}$ \\
\hline (A1) & & $\begin{array}{l}\left\|u-u_{h}\right\|_{1, \Omega} \lesssim h^{s-1}|u|_{s, \Omega} \\
\text { with } 3 / 2<s \leq k+1\end{array}$ \\
\hline$(\mathrm{A} 1+\mathrm{A} 2)$ & $\begin{array}{l}\left\|u-u_{h}\right\|_{1, \Omega} \lesssim c(h) h^{s-1}|u|_{s, \Omega} \\
c(h)=\max _{E \in \Omega_{h}}\left(\log \left(1+\frac{h_{E}}{h_{m(E)}}\right)\right) \\
\text { with } 1<s \leq k+1\end{array}$ & same as for (A1) \\
\hline$(\mathrm{A} 1+\mathrm{A} 3)$ & $\begin{array}{l}\left\|u-u_{h}\right\|_{1, \Omega} \lesssim h^{s-1}|u|_{s, \Omega} \\
\text { with } 1<s \leq k+1\end{array}$ & same as for (A1) \\
\hline
\end{tabular}

in order to make the notation shorter, for any non-negative real $s$ we will denote by

$$
\|v\|_{s, \omega}=\|v\|_{H^{s}(\omega)}, \quad|v|_{s, \omega}=|v|_{H^{s}(\omega)},
$$

the standard $H^{s}$-Sobolev (semi)norm on the measurable open set $\omega$ (see for instance Ref. 3). In particular, the $H^{1 / 2}$-boundary seminorm will have an important role in the paper. We thus recall its definition:

$$
|v|_{1 / 2, \partial E}^{2}:=\int_{\partial E} \int_{\partial E}\left(\frac{v\left(s_{1}\right)-v\left(s_{2}\right)}{s_{1}-s_{2}}\right)^{2} d s_{1} d s_{2},
$$

where, with a small abuse of notation, $v$ stands for $\left.v\right|_{\partial E}$, and where $s_{1}, s_{2}$ denote curvilinear abscissae along the boundary.

The main results of this paper are the convergence estimates for Problem 2.13) under different combinations of mesh assumptions and for the two choices detailed in (2.9), (2.10). We summarize them in Table 1 (see also Remark 2.3 below).

The proofs of these results are based on a non-trivial extension of the stability framework developed in Ref. 4, where the constants involved in the stability estimates are carefully tracked. This is performed in Sec. 3

Remark 2.3. An interesting point considered in this paper is the possibility to completely neglect the internal part of the stability form. In other words, we will show that the choice

$$
s_{E}^{\circ}\left(v_{h}, w_{h}\right)=0,
$$

does not spoil the stability feature of the numerical scheme, see Sec. 3.1.

\section{A General Error Analysis}

In this section, we derive an error analysis, more general than the standard one detailed in Ref. 4. We remark that the present approach can be applied to any other linear symmetric elliptic problem. 
For the analysis, the following discrete semi-norm, induced by the stability term, will play an important role:

$$
\|v\|_{E}^{2}:=s_{E}((I-\mathcal{R}) v,(I-\mathcal{R}) v)+a_{E}\left(\Pi_{E} v, \Pi_{E} v\right) \quad \forall v \in V_{E}+\mathcal{V}_{E} .
$$

Above, $\mathcal{V}_{E} \subseteq V_{\mid E}$ is a subspace of sufficiently regular functions for $s_{E}(\cdot, \cdot)$ to make sense. We now introduce the following assumption, for all $E \in \mathcal{T}_{h}$.

Main assumption - We assume that it holds

$$
\begin{aligned}
a_{E}\left(v_{h}, v_{h}\right) & \leq C_{1}(E)\left\|v_{h}\right\|_{E}^{2} \quad \forall v_{h} \in V_{E}, \\
\|p\|_{E}^{2} & \leq C_{2}(E) a_{E}(p, p) \quad \forall p \in \mathbb{P}_{k}(E),
\end{aligned}
$$

with $C_{1}(E), C_{2}(E)$ positive constants which depend on the shape and possibly on the size of $E$.

Differently than the standard analysis of Ref. 4 where a kind of bound (3.3) is assumed for every $v_{h} \in V_{E}$, we here require the estimate only for the polynomials $p \in \mathbb{P}_{k}(E)$. Thus, even when $C_{1}(E)$ and $C_{2}(E)$ can be chosen independent of $E$, on $V_{E}$ the semi-norm induced by the stabilization term may be stronger than the energy $a_{E}(\cdot, \cdot)^{1 / 2}$. An instance of such an occurrence is given in Sec. 4.2, See also Remark 3.1 below.

We start proving the following lemma.

Lemma 3.1. Under assumptions (3.2), (3.3), the local discrete bilinear form (2.11) satisfies the stability condition

$$
C_{\star}(E)\left\|v_{h}\right\|_{E}^{2} \lesssim a_{E}^{h}\left(v_{h}, v_{h}\right) \lesssim C^{\star}(E)\left\|v_{h}\right\|_{E}^{2} \quad \forall v_{h} \in V_{E},
$$

where $C_{\star}(E)=\min \left\{1, C_{2}(E)^{-1}\right\}$ and $C^{\star}(E)=\max \left\{1, C_{1}(E) C_{2}(E)\right\}$. Furthermore, the bound

$$
a_{E}^{h}\left(v_{h}, v_{h}\right) \lesssim\left(1+C_{2}(E)\right)\left(\left\|v_{h}\right\|_{E}^{2}+\left|v_{h}\right|_{1, E}^{2}\right) \quad \forall v_{h} \in V_{E}
$$

holds true.

Proof. We first note that from definition (2.5) it is immediate to check that

$$
\mathcal{R}\left(I-\Pi_{E}\right) v_{h}=0 \quad \forall v_{h} \in V_{E} .
$$

Using first (3.6), then noting that $\Pi_{E}\left(I-\Pi_{E}\right)=0$ and applying (3.3), we obtain (cf. (3.1) ):

$$
\begin{aligned}
a_{E}^{h}\left(v_{h}, v_{h}\right)= & a_{E}\left(\Pi_{E} v_{h}, \Pi_{E} v_{h}\right)+s_{E}\left((I-\mathcal{R})\left(I-\Pi_{E}\right) v_{h},(I-\mathcal{R})\left(I-\Pi_{E}\right) v_{h}\right) \\
= & a_{E}\left(\Pi_{E} v_{h}, \Pi_{E} v_{h}\right)+a_{E}\left(\Pi_{E}\left(I-\Pi_{E}\right) v_{h}, \Pi_{E}\left(I-\Pi_{E}\right) v_{h}\right) \\
& +s_{E}\left((I-\mathcal{R})\left(I-\Pi_{E}\right) v_{h},(I-\mathcal{R})\left(I-\Pi_{E}\right) v_{h}\right) \\
\geq & C_{2}(E)^{-1}\left\|\Pi_{E} v_{h}\right\|_{E}^{2}+\left\|v_{h}-\Pi_{E} v_{h}\right\|_{E}^{2} \geq C_{\star}(E)\left\|v_{h}\right\|_{E}^{2}
\end{aligned}
$$


for all $v_{h} \in V_{E}$, with $C_{\star}(E)=\frac{1}{2} \min \left\{1, C_{2}(E)^{-1}\right\}$. Again using the first identity in (3.7), recalling definition (3.1), from the triangle inequality we get

$$
a_{E}^{h}\left(v_{h}, v_{h}\right) \leq\left\|v_{h}\right\|_{E}^{2}+\left\|v_{h}-\Pi_{E} v_{h}\right\|_{E}^{2} \leq 3\left\|v_{h}\right\|_{E}^{2}+2\left\|\Pi_{E} v_{h}\right\|_{E}^{2} .
$$

Since $\Pi_{E}$ is a projection with respect to $a^{E}$ and using 3.3 we obtain

$$
\left\|\Pi_{E} v_{h}\right\|_{E}^{2} \leq C_{2}(E) a_{E}\left(\Pi_{E} v_{h}, \Pi_{E} v_{h}\right) \leq C_{2}(E) a_{E}\left(v_{h}, v_{h}\right) .
$$

From (3.9) we immediately get

$$
\left\|\Pi_{E} v_{h}\right\|_{E}^{2} \leq C_{2}(E) M\left|v_{h}\right|_{1, E}^{2},
$$

and also, recalling (3.2),

$$
\left\|\Pi_{E} v_{h}\right\|_{E}^{2} \leq C_{1}(E) C_{2}(E)\left\|v_{h}\right\|_{E}^{2} .
$$

Combining the above bounds it follows:

$$
\begin{aligned}
& a_{E}^{h}\left(v_{h}, v_{h}\right) \leq 3\left\|v_{h}\right\|_{E}^{2}+2 C_{2}(E) M\left|v_{h}\right|_{1, E}^{2}, \\
& a_{E}^{h}\left(v_{h}, v_{h}\right) \leq C^{\star}(E)\left\|v_{h}\right\|_{E}^{2}
\end{aligned}
$$

with $C^{\star}(E)=3+2 C_{1}(E) C_{2}(E)$.

As an immediate consequence of Lemma 3.1] and [3.2), the discrete bilinear form 2.12) associated to 2.11 satisfies

$$
a^{h}\left(v_{h}, v_{h}\right) \geq C_{\text {stab }}(h) a\left(v_{h}, v_{h}\right) \geq C_{\text {stab }}(h) \alpha\left\|v_{h}\right\|_{1, \Omega}^{2} \quad \forall v_{h} \in V_{h},
$$

where

$$
C_{\text {stab }}(h)=\min _{E \in \mathcal{T}_{h}} \frac{C_{\star}(E)}{C_{1}(E)} .
$$

Therefore, due to (2.2), the discrete problem is positive definite and problem (2.13) has a unique solution. For all sufficiently regular functions $v$, we now introduce the global semi-norms

$$
\|v\|^{2}=\sum_{E \in \mathcal{T}_{h}}\|v\|_{E}^{2}, \quad|v|_{1, h}^{2}=\sum_{E \in \mathcal{T}_{h}}|v|_{1, E}^{2}
$$

We notice that, by (3.2) and the Poincaré inequality, $\|\cdot\|$ is a norm on $V_{h}$, not only a semi-norm. Furthermore, for any $h$, let $\mathfrak{F}_{h}$ denote the quantity

$$
\mathfrak{F}_{h}=\sup _{v_{h} \in V_{h}} \frac{\left(f, v_{h}\right)-\left\langle f_{h}, v_{h}\right\rangle}{\left\|v_{h}\right\|} .
$$

We remark that, again by (3.2), it holds:

$$
\mathfrak{F}_{h} \lesssim \sup _{v_{h} \in V_{h}} \frac{\left(f, v_{h}\right)-\left\langle f_{h}, v_{h}\right\rangle}{\left|v_{h}\right|_{1, \Omega}}
$$

Therefore, taking $f_{h}$ as in Ref. 4 and using the arguments in that paper, we infer:

$$
\mathfrak{F}_{h} \leq C(f) h^{k},
$$

where $C(f)$ depends on suitable Sobolev norms of the source term $f$. 
We have the following result, stating a kind of generalized best-approximation property (see also Remark 3.1 below).

Theorem 3.2. Let assumptions (3.2) and (3.3) hold. Let the continuous solution of (2.1) satisfy $u_{\mid E} \in \mathcal{V}_{E}$ for all $E \in \mathcal{T}_{h}$, where $\mathcal{V}_{E} \subseteq V_{\mid E}$ is a subspace of sufficiently regular functions for $s_{E}(\cdot, \cdot)$ to make sense. Then, for every $u_{I} \in V_{h}$ and for every $u_{\pi}$ such that $u_{\pi \mid E} \in \mathbb{P}_{k}(E)$, the discrete solution $u_{h}$ of (2.13) with bilinear form (2.11) satisfies

$$
\left|u-u_{h}\right|_{1, \Omega} \lesssim C_{\text {err }}(h)\left(\left(\mathfrak{F}_{h}\right)+\left\|u-u_{I}\right\|+\left\|u-u_{\pi}\right\||+| u-\left.u_{I}\right|_{1, \Omega}+\left|u-u_{\pi}\right|_{1, h}\right) .
$$

Setting

$$
\begin{aligned}
\widetilde{C}(h) & =\max _{E \in \mathcal{T}_{h}}\left\{1, C_{2}(E)\right\}, \quad C_{1}(h)=\max _{E \in \mathcal{T}_{h}}\left\{C_{1}(E)\right\}, \\
C^{\star}(h) & =\max _{E \in \mathcal{T}_{h}}\left\{C^{\star}(E)\right\},
\end{aligned}
$$

the constant $C_{\mathrm{err}}(h)$ is given by

$$
C_{\mathrm{err}}(h)=\max \left\{1, \widetilde{C}(h) C_{1}(h), \widetilde{C}(h)^{3 / 2} \sqrt{C^{\star}(h) C_{1}(h)}\right\} .
$$

Proof. First using the coercivity property in Lemma 3.1 then with identical calculations as in Ref. 4 (precisely: Theorem 3.1, Eq. (3.11)), we get

$$
\left\|u_{h}-u_{I}\right\|^{2} \leq \widetilde{C}(h) a^{h}\left(u_{h}-u_{I}, u_{h}-u_{I}\right)=\widetilde{C}(h)\left(T_{1}+T_{2}+T_{3}\right),
$$

where $\widetilde{C}(h)=\max _{E \in \mathcal{T}_{h}}\left\{1, C_{2}(E)\right\}$, and the terms $T_{i}$ are given by

$$
\begin{aligned}
& T_{1}=\left\langle f_{h}, u_{h}-u_{I}\right\rangle-\left(f, u_{h}-u_{I}\right), \\
& T_{2}=\sum_{E \in \mathcal{T}_{h}} a_{E}^{h}\left(u_{\pi}-u_{I}, u_{h}-u_{I}\right), \\
& T_{3}=\sum_{E \in \mathcal{T}_{h}} a_{E}\left(u-u_{\pi}, u_{h}-u_{I}\right) .
\end{aligned}
$$

For term $T_{1}$, definition (3.13) and assumption (3.2) yield

$$
T_{1} \lesssim \mathfrak{F}_{h}\left|u_{h}-u_{I}\right|_{1, \Omega} \lesssim \sqrt{C_{1}(h)} \mathfrak{F}_{h}\left\|u_{h}-u_{I}\right\|
$$

where $C_{1}(h)=\max _{E \in \mathcal{T}_{h}}\left\{C_{1}(E)\right\}$. Term $T_{2}$ is treated using both the bounds (3.4) and (3.5), that easily lead to the estimate

$$
\begin{aligned}
T_{2} & \lesssim \sqrt{C^{\star}(h) \widetilde{C}(h)}\left(\left\|u_{\pi}-u_{I}\right\|+\left|u_{\pi}-u_{I}\right|_{1, \Omega}\right)\left\|u_{h}-u_{I}\right\| \\
& \leq \sqrt{C^{\star}(h) \widetilde{C}(h)}\left(\left\|u-u_{I}\right\|+\left\|u-u_{\pi}\right\|+\left|u-u_{I}\right|_{1, \Omega}+\left|u-u_{\pi}\right|_{1, h}\right)\left\|u_{h}-u_{I}\right\|,
\end{aligned}
$$


where $C^{\star}(h)=\max _{E \in \mathcal{T}_{h}}\left\{C^{\star}(E)\right\}$. Term $T_{3}$ is bounded using the piecewise continuity in $H^{1}$ of the continuous bilinear form and (3.2):

$$
\begin{aligned}
T_{3} & \lesssim \sqrt{C_{1}(h)} \sum_{E \in \mathcal{T}_{h}}\left|u-u_{\pi}\right|_{1, E}\left\|u_{h}-u_{I}\right\|_{E} \\
& \leq \sqrt{C_{1}(h)}\left|u-u_{\pi}\right|_{1, h}\left\|u_{h}-u_{I}\right\| .
\end{aligned}
$$

From (3.18), using the bounds (3.19), (3.20) and (3.21), then dividing by the term $\left\|u_{h}-u_{I}\right\|$, we get

$$
\begin{aligned}
\left\|u_{h}-u_{I}\right\| \lesssim & \widetilde{C}(h) \max \left\{\sqrt{C_{1}(h)}, \sqrt{C^{\star}(h) \widetilde{C}(h)}\right\} \\
& \times\left(\mathfrak{F}_{h}+\left\|u-u_{I}\right\|+\left\|u-u_{\pi}\right\|+\left|u-u_{I}\right|_{1, \Omega}+\left|u-u_{\pi}\right|_{1, h}\right) .
\end{aligned}
$$

The triangle inequality and (3.2) give

$$
\begin{aligned}
\left|u-u_{h}\right|_{1, \Omega} & \leq\left|u-u_{I}\right|_{1, \Omega}+\left|u_{h}-u_{I}\right|_{1, \Omega} \\
& \leq\left|u-u_{I}\right|_{1, \Omega}+\sqrt{C_{1}(h)}\left\|u_{h}-u_{I}\right\|,
\end{aligned}
$$

Combining (3.22) and (3.23), we get (3.16) with

$$
C_{\mathrm{err}}(h)=\max \left\{1, \widetilde{C}(h) C_{1}(h), \widetilde{C}(h)^{3 / 2} \sqrt{C^{\star}(h) C_{1}(h)}\right\} .
$$

Corollary 3.3. By using (3.22) and the triangle inequality it is immediate to check that, as a corollary of the above result, it also holds

$$
\left\|u-u_{h}\right\| \leq \widetilde{C}_{\mathrm{err}}(h)\left(\left(\mathfrak{F}_{h}\right)+\left\|u-u_{I}\right\|+\left\|u-u_{\pi}\right\|+\left|u-u_{\pi}\right|_{1, h}\right),
$$

where $\widetilde{C}_{\mathrm{err}}(h)=\max \left\{1, \widetilde{C}(h) \sqrt{C_{1}(h)}, \widetilde{C}(h)^{3 / 2} \sqrt{C^{\star}(h)}\right\}$.

Remark 3.1. In most cases of interest, the constants in (3.2) and (3.3) are in fact either mildly dependent on, or even independent of the size of $E$ (examples will be shown in the sequel). In this latter instance, the constant $C_{\text {err }}(h)$ in (3.16) is $O(1)$, thus recovering a typical error estimate for Galerkin schemes.

We conclude this subsection with an extension of the approximation result in Ref. 27 to the case of higher-order norms and more general mesh assumptions.

Theorem 3.4. Let assumption (A1) hold. Then there exist a real number $\bar{\sigma}>3 / 2$ and a linear operator $\mathcal{I}_{h}: H^{s}(\Omega) \rightarrow V_{h}$, with $1<s \leq k+1$, such that it holds:

$$
\left|u-\mathcal{I}_{h} u\right|_{\sigma, E} \lesssim h_{E}^{s-\sigma}|u|_{s, E}, \quad E \in \Omega_{h}, \quad u \in H^{s}(\Omega), \quad \text { and } \quad 1 \leq \sigma<\min \{\bar{\sigma}, s\} .
$$

Proof. For each element $E$, we can build a sub-triangulation by connecting all its vertices with the center $\mathbf{x}_{E}$ introduced in assumption (A1). We denote by $\mathcal{T}_{h}$ the global (conforming) triangular mesh obtained by applying such a procedure for all $E \in \Omega_{h}$. It is easy to check that, under assumption (A1), the triangles in the 
sequence of meshes $\left\{\mathcal{T}_{h}\right\}_{h}$ have maximum angles that are uniformly bounded away from $\pi$ (although shape regularity is not guaranteed).

Let $u_{r}$ be the standard continuous and piecewise $\mathbb{P}_{k}$ polynomial Lagrange interpolant of $u$ over the triangulation $\mathcal{T}_{h}$. Then it holds:

$$
\left|u-u_{r}\right|_{\sigma, E} \lesssim h_{E}^{s-\sigma}|u|_{s, E}, \quad E \in \Omega_{h}, \quad 1 \leq \sigma<s \leq k+1, \quad u \in H^{s}(\Omega)
$$

where we used the anisotropic approximation results in Ref. 2 also recalling the angle property above. In the following, we denote by $u_{\pi}$ a piecewise discontinuous polynomial approximation of $u$ over the mesh $\Omega_{h}$. For instance, one may think of the $L^{2}$-projection of $u$ on $\mathbb{P}_{k}(E)$ for each element $E$.

We now introduce the function $\mathcal{I}_{h} u \in V_{h}$ defined, on each element $E$, by

$$
\begin{cases}\Delta\left(\mathcal{I}_{h} u\right)=\Delta u_{\pi} & \text { in } E, \\ \mathcal{I}_{h} u=u_{r} & \text { on } \partial E,\end{cases}
$$

so that $\left(\mathcal{I}_{h} u-u_{\pi}\right)$ satisfies on every $E$

$$
\begin{cases}\Delta\left(\mathcal{I}_{h} u-u_{\pi}\right)=0 & \text { in } E, \\ \mathcal{I}_{h} u-u_{\pi}=u_{r}-u_{\pi} & \text { on } \partial E .\end{cases}
$$

Therefore, for all $E \in \Omega_{h}$, regularity results on Lipschitz domains (see Ref. 25) guarantee that

$$
\left|\mathcal{I}_{h} u-u_{\pi}\right|_{\sigma, E} \lesssim\left|u_{r}-u_{\pi}\right|_{\sigma-1 / 2, \partial E} \quad 1 \leq \sigma \leq \bar{\sigma}_{E}
$$

where $\bar{\sigma}_{E}=2$ if $E$ is convex, and $\bar{\sigma}_{E}=1+\pi / \omega_{E}$ (with $\omega_{E}$ the largest angle of $E)$ otherwise. Let now $\bar{\sigma}=\min _{E \in\left\{\Omega_{h}\right\}_{h}} \bar{\sigma}_{E}$, where we stress that the minimum is taken among all elements of the whole mesh sequence. Due to assumption (A1), that yields a uniform bound on the maximum element angles, the number $\bar{\sigma}$ is strictly bigger than 3/2. First a triangle inequality and bound (3.27), then a trace inequality yield

$$
\begin{aligned}
\left|u-\mathcal{I}_{h} u\right|_{\sigma, E} & \lesssim\left|u-u_{\pi}\right|_{\sigma, E}+\left|u_{r}-u_{\pi}\right|_{\sigma-1 / 2, \partial E} \lesssim\left|u-u_{\pi}\right|_{\sigma, E}+\left|u_{r}-u_{\pi}\right|_{\sigma, E} \\
& \lesssim\left|u-u_{\pi}\right|_{\sigma, E}+\left|u-u_{r}\right|_{\sigma, E}
\end{aligned}
$$

for all $1 \leq \sigma \leq \bar{\sigma}$ and all $E \in \Omega_{h}$. The result follows combining the above bound with (3.25) and standard polynomial approximation estimates on shape regular polygons.

\subsection{Reduction to the boundary}

In this section, we derive a result that allows to focus the analysis of assumptions (3.2) and (3.3) only on the boundary of the element. The boundary bilinear form $s_{E}^{\partial}(\cdot, \cdot)$ is here one of the two detailed in (2.9) and (2.10), but the same analysis applies to different choices, such as the ones outlined in Remark 4.1. We start by showing the following lemma. 
Lemma 3.5. For all $v_{h} \in V_{E}$, there exists a polynomial $\tilde{p} \in \mathbb{P}_{k}(E)$ such that $\Delta \widetilde{p}=\Delta v_{h}$ satisfying:

$$
|\widetilde{p}|_{1, E} \lesssim h_{E}\left\|\Delta v_{h}\right\|_{0, E}
$$

Proof. We only sketch the very simple proof. Since for all $v_{h} \in V_{E}$ it holds $\Delta v_{h} \in$ $\mathbb{P}_{k-2}(E)$, there are (infinitely many) polynomials of degree $k$ that satisfy $\Delta \widetilde{p}=\Delta v_{h}$ (cf. Ref. 30, for instance). In order to derive the bound (B.28), we first note that, thanks to assumption (A1) and since $\Delta \widetilde{p}=\Delta v_{h}$, inequality (3.28) is equivalent to

$$
|\widetilde{p}|_{1, B_{E}} \lesssim h_{E}\|\Delta \widetilde{p}\|_{0, B_{E}} .
$$

The above bound, that is now restricted on balls, can be easily deduced by choosing $\widetilde{p}$ in the subspace

$$
\left\{q \in \mathbb{P}_{k}\left(B_{E}\right): \int_{B_{E}} q p=0 \text { for all harmonic polynomials } p \in \mathbb{P}_{k}\left(B_{E}\right)\right\}
$$

and by a scaling argument.

Concerning assumption (3.2), we have the following result.

Proposition 3.6. Let assumption (A1) and let $s_{E}^{\circ}(\cdot, \cdot)$ be given as in (2.8). Assume the existence of a positive constant $\widehat{C}_{1}(E)$ such that

$$
\left|v_{h}\right|_{1 / 2, \partial E}^{2} \leq \widehat{C}_{1}(E)\left(s_{E}^{\partial}\left((I-\mathcal{R}) v_{h},(I-\mathcal{R}) v_{h}\right)+\left|\Pi_{E} v_{h}\right|_{1, E}^{2}\right) \quad \forall v_{h} \in V_{E} .
$$

Then assumption (3.2) holds with $C_{1}(E) \lesssim \max \left\{1, \widehat{C}_{1}(E)\right\}$.

Proof. Let $v_{h} \in V_{E}$ and $\widetilde{p}$ as in Lemma 3.5 Let $\bar{v}_{h}$ be the unique constant function on $E$ such that $\int_{\partial E} \bar{v}_{h}=\int_{\partial E} v_{h}$. Then, first by an integration by parts and then by the definition of $\Pi_{E}$, we get

$$
\begin{aligned}
\int_{E}\left(v_{h}-\bar{v}_{h}\right) \Delta v_{h} d x & =\int_{E}\left(v_{h}-\bar{v}_{h}\right) \Delta \widetilde{p} d x \\
& =-\int_{E} \nabla v_{h} \cdot \nabla \widetilde{p} d x+\int_{\partial E}\left(v_{h}-\bar{v}_{h}\right)\left(\nabla \widetilde{p} \cdot \mathbf{n}_{E}\right) d s \\
& =-\int_{E} \nabla \Pi_{E} v_{h} \cdot \nabla \widetilde{p} d x+\int_{\partial E}\left(v_{h}-\bar{v}_{h}\right)\left(\nabla \widetilde{p} \cdot \mathbf{n}_{E}\right) .
\end{aligned}
$$

Again an integration by parts and (3.30) yield

$$
\begin{aligned}
a_{E}\left(v_{h}, v_{h}\right) & \lesssim\left|v_{h}\right|_{1, E}^{2}=\left|\left(v_{h}-\bar{v}_{h}\right)\right|_{1, E}^{2} \\
& =-\int_{E}\left(v_{h}-\bar{v}_{h}\right) \Delta v_{h} d x+\int_{\partial E}\left(v_{h}-\bar{v}_{h}\right)\left(\nabla v_{h} \cdot \mathbf{n}_{E}\right) d s \\
& =\int_{E} \nabla \Pi_{E} v_{h} \cdot \nabla \widetilde{p} d x+\int_{\partial E}\left(v_{h}-\bar{v}_{h}\right)\left(\nabla\left(v_{h}-\widetilde{p}\right) \cdot \mathbf{n}_{E}\right) d s \\
& =T_{1}+T_{2}
\end{aligned}
$$


with $\mathbf{n}_{E}$ denoting the outward unit normal to the boundary of $E$. The first term above is bounded by the Cauchy-Schwarz inequality, and by a combination of Lemma 3.5 with the estimate (see Lemma 6.3):

$$
\left\|\Delta v_{h}\right\|_{0, E} \lesssim h_{E}^{-1}\left|v_{h}\right|_{1, E} \quad \forall v_{h} \in V_{E}
$$

We thus obtain

$$
T_{1} \leq\left|\Pi_{E} v_{h}\right|_{1, E}|\widetilde{p}|_{1, E} \lesssim\left|\Pi_{E} v_{h}\right|_{1, E}\left|v_{h}\right|_{1, E} \lesssim\left\|v_{h}\right\| \|_{E}\left|v_{h}\right|_{1, E}
$$

For the second term, we first note that $\operatorname{div}\left(\nabla\left(v_{h}-\widetilde{p}\right)\right)=\Delta\left(v_{h}-\widetilde{p}\right)=0$. Therefore, after applying a (scaled) duality bound on the boundary of $E$, we can use the estimate (see Lemma 6.2):

$$
\left|\mathbf{w} \cdot \mathbf{n}_{E}\right|_{H^{-1 / 2}(\partial E)} \lesssim\|\mathbf{w}\|_{0, E} \quad \forall \mathbf{w} \in\left[L^{2}(E)\right]^{2} \quad \text { s.t. } \operatorname{div} \mathbf{w}=0
$$

with $\mathbf{w}=\nabla\left(v_{h}-\tilde{p}\right)$, and where $H^{-1 / 2}(\partial E)$ denotes the dual space of $H^{1 / 2}(\partial E)$. Thus, we obtain

$$
\begin{aligned}
\left|T_{2}\right| & \lesssim\left(\left|\left(v_{h}-\bar{v}_{h}\right)\right|_{1 / 2, \partial E}+h_{E}^{-1 / 2}\left\|\left(v_{h}-\bar{v}_{h}\right)\right\|_{0, \partial E}\right)\left\|\nabla\left(v_{h}-\widetilde{p}\right) \cdot \mathbf{n}_{E}\right\|_{H^{-1 / 2}(\partial E)} \\
& \lesssim\left(\left|v_{h}\right|_{1 / 2, \partial E}+h_{E}^{-1 / 2}\left\|\left(v_{h}-\bar{v}_{h}\right)\right\|_{0, \partial E}\right)\left|\left(v_{h}-\widetilde{p}\right)\right|_{1, E} .
\end{aligned}
$$

Moreover, by standard approximation estimates in one dimension, it holds $h_{E}^{-1 / 2}\left\|\left(v_{h}-\bar{v}_{h}\right)\right\|_{0, \partial E} \lesssim\left|v_{h}\right|_{1 / 2, \partial E}$. Therefore, using (3.29), the triangle inequality and again Lemma 3.5 with 3.32, bound 3.35 yields

$$
\begin{aligned}
\left|T_{2}\right| & \left.\left.\lesssim\left|v_{h}\right|_{1 / 2, \partial E}\left(\left|v_{h}\right|_{1, E}+\mid \widetilde{p}\right)\right|_{1, E}\right) \\
& \leq \widehat{C}_{1}(E)\left(s_{E}^{\partial}\left((I-\mathcal{R}) v_{h},(I-\mathcal{R}) v_{h}\right)+\left|\Pi_{E} v_{h}\right|_{1, E}^{2}\right)\left|v_{h}\right|_{1, E} \\
& \leq \widehat{C}_{1}(E)\left\|v_{h}\right\|_{E}\left|v_{h}\right|_{1, E} .
\end{aligned}
$$

The result follows by combining Eqs. 3.31, 3.33), 3.36) and recalling that $\left|v_{h}\right|_{1, E}^{2} \lesssim a_{E}\left(v_{h}, v_{h}\right)$.

Furthermore, concerning assumption (3.3), we have the following result.

Proposition 3.7. Let assumption (A1) hold and let $s_{E}^{\circ}(\cdot, \cdot)$ as given in (2.8). Assume the existence of a positive constant $\widehat{C}_{2}(E)$ such that

$$
s_{E}^{\partial}((I-\mathcal{R}) p,(I-\mathcal{R}) p) \leq \widehat{C}_{2}(E)|p|_{1, E}^{2} \quad \forall p \in \mathbb{P}_{k}(E) .
$$

Then assumption (3.3) holds with $C_{2}(E) \lesssim \max \left\{1, \widehat{C}_{2}(E)\right\}$.

Proof. We first note that the second term in (3.1) is immediately bounded:

$$
a_{E}\left(\Pi_{E} p, \Pi_{E} p\right)=a_{E}(p, p) \leq M|p|_{1, E}^{2} \quad \forall p \in \mathbb{P}_{k}(E) .
$$


Therefore, by the definition of $s_{E}(\cdot, \cdot)$ and using (3.37), it is sufficient to show that

$$
s_{E}^{\circ}((I-\mathcal{R}) p,(I-\mathcal{R}) p) \lesssim|p|_{1, E}^{2} \quad \forall p \in \mathbb{P}_{k}(E) .
$$

By definition of $s_{E}^{\circ}(\cdot, \cdot)$ and recalling that $\left\|m_{i}\right\|_{L^{\infty}(E)} \lesssim 1, i=1,2, \ldots, n_{k-2}$, we have

$$
\begin{aligned}
s_{E}^{\circ}((I-\mathcal{R}) p,(I-\mathcal{R}) p) & =\sum_{i=1}^{n_{k-2}} \Xi_{i}^{\circ}((I-\mathcal{R}) p)^{2} \\
& =\sum_{i=1}^{n_{k-2}}|E|^{-2}\left(\int_{E}((I-\mathcal{R}) p) m_{i}\right)^{2} \\
& \lesssim \sum_{i=1}^{n_{k-2}}|E|^{-1}\|(I-\mathcal{R}) p\|_{0, E}^{2} .
\end{aligned}
$$

We now notice that, under assumption (A1) we have:

$$
\|v-\mathcal{R} v\|_{0, E} \lesssim h_{E}|v|_{1, E} \quad \forall v \in H^{1}(E)
$$

that follows easily from standard approximation theory on shape regular polygons. Using (3.41) and (3.40), we obtain

$$
s_{E}^{\circ}((I-\mathcal{R}) p,(I-\mathcal{R}) p) \lesssim \sum_{i=1}^{n_{k-2}}|p|_{H^{1}(E)}^{2} \lesssim|p|_{1, E}^{2} .
$$

Remark 3.2. Interestingly enough, we observe that Propositions 3.6 and 3.7 remain true if we completely neglect $s_{E}^{\circ}(\cdot, \cdot)$. Indeed, if $s_{E}^{\circ}(\cdot, \cdot)=0$, the proof of Proposition 3.6 applies identically while the proof of Proposition 3.7 gets simpler, since showing (3.39) becomes trivial. Therefore, selecting $s_{E}^{\circ}(v, w)=0$ instead of (2.8), cf. Remark 2.3, does not spoil the stability features of the method.

\section{Analysis of Two Choices for the Boundary Stabilization}

In this section, we apply Propositions 3.6 and 3.7 for a couple of classical choices of the boundary stability term $s_{E}^{\partial}(\cdot, \cdot)$, see (2.9) and (2.10). Compared to the theory presented in Ref. 4 this allows to relax the mesh assumptions in establishing stability and convergence properties of the proposed methods.

\subsection{Identity matrix: Choice (2.9)}

This is the more classical, and simpler to code, choice for virtual elements. We recall it here again, for convenience:

$$
s_{E}^{\partial}\left(v_{h}, w_{h}\right)=\sum_{i=1}^{N k} \Xi_{i}^{\partial}\left(v_{h}\right) \Xi_{i}^{\partial}\left(w_{h}\right) .
$$


We may call it the identity matrix choice since in the implementation procedure of the method, the bilinear form (4.1) is clearly associated with an identity matrix of dimension $N k$. We have the following stability result.

Proposition 4.1. Let assumptions (A1) and (A2) hold. Then, for the boundary form 4.1), conditions (3.29) and (3.37) hold with positive constants $\widehat{C}_{1}$ and $\widehat{C}_{2}$ that satisfy

$$
\widehat{C}_{1}(E) \lesssim\left(\log \left(1+h_{E} / h_{m(E)}\right)\right), \quad \widehat{C}_{2}(E) \lesssim 1
$$

where $h_{m(E)}$ denotes the length of the smallest edge in $E$.

Proof. Standard results for polynomials in one dimension immediately give

$$
\left\|w_{h}\right\|_{L^{\infty}(\partial E)}^{2} \lesssim s_{E}^{\partial}\left(w_{h}, w_{h}\right), \quad \forall w_{h} \in V_{E}
$$

We now use the estimate (proved in Lemma 6.6):

$$
\forall w_{h} \in V_{E}:\left|w_{h}\right|_{1 / 2, \partial E}^{2} \lesssim \widehat{C}(E)\left\|w_{h}\right\|_{L^{\infty}(\partial E)}^{2}, \quad \widehat{C}(E)=\left(\log \left(1+h_{E} / h_{m(E)}\right)\right)
$$

In combination with (4.3), it yields:

$$
\begin{aligned}
\left|v_{h}\right|_{1 / 2, \partial E}^{2} & =\left|v_{h}-\mathcal{R} v_{h}\right|_{1 / 2, \partial E}^{2} \lesssim \widehat{C}(E) s_{E}^{\partial}\left((I-\mathcal{R}) v_{h},(I-\mathcal{R}) v_{h}\right) \\
& \left.\lesssim \widehat{C}(E)\left(s_{E}^{\partial}\left((I-\mathcal{R}) v_{h},(I-\mathcal{R}) v_{h}\right)+\left|\Pi_{E} v_{h}\right|_{1, E}^{2}\right)\right) \quad \forall v_{h} \in V_{E}
\end{aligned}
$$

i.e. condition (3.29) holds with $\widehat{C}_{1}(E) \lesssim\left(\log \left(1+h_{E} / h_{m(E)}\right)\right)$.

We now prove that estimate (3.37) holds. Recalling assumption (A2), it is immediate to check that

$$
s_{E}^{\partial}(v, v) \lesssim N\|v\|_{L^{\infty}(\partial E)}^{2} \lesssim\|v\|_{L^{\infty}(\partial E)}^{2} \quad \forall v \in C^{0}(\partial E)
$$

Take any $p \in \mathbb{P}_{k}(E)$. We get, using bound (4.6), an inverse estimate for polynomials (cf. Remark 6.1), and recalling (3.41):

$$
\begin{aligned}
s_{E}^{\partial}((I-\mathcal{R}) p,(I-\mathcal{R}) p) & \lesssim\|(I-\mathcal{R}) p\|_{L^{\infty}(\partial E)}^{2} \lesssim\|(I-\mathcal{R}) p\|_{L^{\infty}(E)}^{2} \\
& \lesssim h_{E}^{-2}\|(I-\mathcal{R}) p\|_{0, E}^{2} \leq|p|_{1, E}^{2},
\end{aligned}
$$

i.e. condition (3.37) holds with $\widehat{C}_{2}(E) \lesssim 1$. 
The following theorem shows that, even in the presence of arbitrarily small edges (provided the number of edges are uniformly bounded), the convergence rate of the VEM is optimal up to a loss of a logarithmic factor.

Theorem 4.2. Let assumptions (A1) and (A2) hold. Let $u$ be the solution of problem (2.1), assumed to be in $H^{s}(\Omega), s>1$. Let $u_{h}$ be the solution of the discrete problem (2.13). Then it holds

$$
\left\|u-u_{h}\right\|_{1, \Omega} \lesssim c(h) h^{s-1}|u|_{s, \Omega} \quad 1<s \leq k+1 .
$$

with

$$
c(h)=\max _{E \in \Omega_{h}}\left(\log \left(1+h_{E} / h_{m(E)}\right)\right) .
$$

If the stronger assumption (A3) holds, then clearly $c(h) \lesssim 1$.

Proof. Proposition 4.1 allows to apply Propositions 3.6 and 3.7. Therefore, assumptions (3.2) and (3.3) hold with $C_{1}(E) \lesssim \log \left(1+h_{E} / h_{m(E)}\right)$ and $C_{2}(E) \lesssim 1$, respectively. Then, Theorem 3.2 can be invoked; a look at the constants shows that

$$
C_{\text {err }}(h) \lesssim 1+\max _{E \in \Omega_{h}}\left\{C_{1}(E)\right\} \lesssim c(h) .
$$

We now estimate the terms in the right-hand side of (3.16). We first recall (3.15):

$$
\mathfrak{F}_{h} \lesssim h^{k}
$$

Moreover, Theorem 3.4 with $u_{I}:=\mathcal{I}_{h} u$ shows that

$$
\left|u-u_{I}\right|_{1, \Omega} \lesssim\left(\sum_{E \in \mathcal{T}_{h}} h_{E}^{2 s-2}|u|_{s, E}^{2}\right)^{1 / 2} \lesssim h^{s-1}|u|_{s, \Omega} \quad 1<s \leq k+1 .
$$

We now choose $u_{\pi}$ as the $L^{2}$-projection of $u$ on $\mathbb{P}_{k}(E)$ for each element $E$. Standard approximation results on polygons (see for instance Ref. 221) yield

$$
\begin{aligned}
\left(\sum_{E \in \mathcal{T}_{h}}\left|u-u_{\pi}\right|_{1, E}^{2}\right)^{1 / 2} & \lesssim\left(\sum_{E \in \mathcal{T}_{h}} h_{E}^{2 s-2}|u|_{s, E}^{2}\right)^{1 / 2} \\
& \lesssim h^{s-1}|u|_{s, \Omega} \quad 1<s \leq k+1 .
\end{aligned}
$$

We now look into the term $\left\|u-u_{I}\right\|$. From (2.7), 3.1 and (3.12), we deduce that we need to estimate:

(1) the term

$$
a_{E}\left(\Pi_{E}\left(u-u_{I}\right), \Pi_{E}\left(u-u_{I}\right)\right) ;
$$

(2) the term

$$
s_{E}^{\partial}\left((I-\mathcal{R})\left(u-u_{I}\right),(I-\mathcal{R})\left(u-u_{I}\right)\right)=\sum_{i=1}^{N k} \Xi_{i}^{\partial}\left((I-\mathcal{R})\left(u-u_{I}\right)\right)^{2}
$$


(3) the term, see (2.8):

$$
s_{E}^{\circ}\left((I-\mathcal{R})\left(u-u_{I}\right),(I-\mathcal{R})\left(u-u_{I}\right)\right)=\sum_{i=1}^{k(k-1) / 2} \Xi_{i}^{\circ}\left((I-\mathcal{R})\left(u-u_{I}\right)\right)^{2} .
$$

Take $s$ with $1<s<k+1$, and $\varepsilon$ such that $0<\varepsilon<\min \{1 / 2, s-1\}$. Regarding (4.11), we notice that from the continuity of $\Pi_{E}$ and from Theorem 3.4 with $\sigma=1$, it holds

$$
a_{E}\left(\Pi_{E}\left(u-u_{I}\right), \Pi_{E}\left(u-u_{I}\right)\right) \lesssim\left|u-u_{I}\right|_{1, E}^{2} \lesssim h_{E}^{2 s-2}|u|_{s, E}^{2} .
$$

Now, the Sobolev embedding $H^{1 / 2+\varepsilon}(\partial E) \subset C^{0}(\partial E)$ shows that it holds:

$$
\|v\|_{L^{\infty}(\partial E)} \lesssim h_{E}^{-1 / 2}\|v\|_{0, \partial E}+h_{E}^{\varepsilon}|v|_{1 / 2+\varepsilon, \partial E} \quad \forall v \in H^{s}(E) .
$$

A scaled trace inequality, that can be derived by an argument analogous to that in Lemma 6.1, gives

$$
h_{E}^{-1 / 2}\|v\|_{0, \partial E}+h_{E}^{\varepsilon}|v|_{1 / 2+\varepsilon, \partial E} \lesssim h_{E}^{-1}\|v\|_{0, E}+h_{E}^{\varepsilon}|v|_{1+\varepsilon, E} \quad \forall v \in H^{s}(E) .
$$

Therefore, (4.6), 4.15) and (4.16) yield

$$
s_{E}^{\partial}(v, v) \lesssim h_{E}^{-2}\|v\|_{0, E}^{2}+h_{E}^{2 \varepsilon}|v|_{1+\varepsilon, E}^{2} \quad \forall v \in H^{s}(E) .
$$

Choosing $v=(I-\mathcal{R})\left(u-u_{I}\right)_{\mid E}$ in (4.17), using (3.41) and noting that $\mid(I-\mathcal{R})(u-$ $\left.u_{I}\right)\left.\right|_{1+\varepsilon, E}=\left|u-u_{I}\right|_{1+\varepsilon, E}$, we obtain:

$$
s_{E}^{\partial}\left((I-\mathcal{R})\left(u-u_{I}\right),(I-\mathcal{R})\left(u-u_{I}\right)\right) \lesssim\left|u-u_{I}\right|_{1, E}^{2}+h_{E}^{2 \varepsilon}\left|u-u_{I}\right|_{1+\varepsilon, E}^{2} .
$$

An application of Theorem 3.4 with $\sigma=1$ (respectively, $\sigma=1+\varepsilon$ ) in the first (respectively, second) term of the right-hand side of 4.18) leads to:

$$
s_{E}^{\partial}\left((I-\mathcal{R})\left(u-u_{I}\right),(I-\mathcal{R})\left(u-u_{I}\right)\right) \lesssim h_{E}^{2 s-2}|u|_{s, E}^{2} .
$$

We now notice that bound (3.40) applies also to $\left(u-u_{I}\right)_{\mid E}$, and not only to polynomials $p \in \mathbb{P}_{k}$. Therefore, by using again (3.41) and Theorem 3.4 one easily gets

$$
\begin{aligned}
s_{E}^{\circ}\left((I-\mathcal{R})\left(u-u_{I}\right),(I-\mathcal{R})\left(u-u_{I}\right)\right) & \lesssim h_{E}^{-2}\left\|(I-\mathcal{R})\left(u-u_{I}\right)\right\|_{0, E}^{2} \\
& \lesssim\left|u-u_{I}\right|_{1, E}^{2} \lesssim h_{E}^{2 s-2}|u|_{s, E}^{2} .
\end{aligned}
$$

Combining (4.14), 4.19) and (4.20), we get

$$
\left\|u-u_{I}\right\| \lesssim\left(\sum_{E \in \mathcal{T}_{h}} h_{E}^{2 s-2}|u|_{s, E}^{2}\right)^{1 / 2} \lesssim h^{s-1}|u|_{s, \Omega}, \quad 1<s \leq k+1 .
$$

By following the same steps and using standard approximation results on polygons (see for instance Ref. 22), we get

$$
\left\|u-u_{\pi}\right\| \lesssim\left(\sum_{E \in \mathcal{T}_{h}} h_{E}^{2 s-2}|u|_{s, E}^{2}\right)^{1 / 2} \lesssim h^{s-1}|u|_{s, \Omega}, \quad 1<s \leq k+1 .
$$

We conclude by collecting estimates (4.8), 4.21), (4.22), 4.9) and (4.10). 


\subsubsection{A "classical" stability bound}

We close this part on the identity matrix choice by proving a stability result more in the spirit of Ref. 4, but still under the more general mesh assumptions considered in this paper. These estimates could be used to prove the same error estimate as in Theorem 4.2, by simply applying the standard theory of Ref. 4 .

Proposition 4.3. Let assumptions (A1) and (A2) hold. Then it holds

$$
s_{E}\left(v_{h}, v_{h}\right) \lesssim a_{E}\left(v_{h}, v_{h}\right) \lesssim c(h) s_{E}\left(v_{h}, v_{h}\right) \quad \forall v_{h} \text { with } \Pi_{E} v_{h}=0,
$$

where

$$
c(h)=\max _{E \in \Omega_{h}} \log \left(1+h_{E} / h_{m(E)}\right) .
$$

If the stronger assumption (A3) holds, then clearly $c(h) \lesssim 1$.

Proof. Proposition 4.1 combined with Proposition 3.6 gives the validity of (3.2) with constant $C_{1}(E) \lesssim c(h)$. Since $\Pi_{E} v_{h}=0$ implies $\mathcal{R} v_{h}=0$, bound (3.2) yields the second inequality in (4.23). We now show the other bound. By definition, the Hölder inequality and recalling $\left\|m_{i}\right\|_{L^{\infty}} \lesssim 1$, we get the estimate:

$$
s_{E}^{\circ}\left(v_{h}, v_{h}\right)=\sum_{i=1}^{n_{k-2}} \Xi_{i}^{\circ}\left(v_{h}\right)^{2}=\sum_{i=1}^{n_{k-2}}|E|^{-2}\left(\int_{E} v_{h} m_{i}\right)^{2} \lesssim \sum_{i=1}^{n_{k-2}}|E|^{-1}\left\|v_{h}\right\|_{0, E}^{2},
$$

for all $v_{h} \in V_{h \mid E}$.

Regarding the term $s_{E}^{\partial}\left(v_{h}, v_{h}\right)$, due to assumption (A2), it is immediate to derive

$$
s_{E}^{\partial}\left(v_{h}, v_{h}\right) \lesssim\left\|v_{h}\right\|_{L^{\infty}(\partial E)}^{2} .
$$

We now use the estimates

$$
\begin{aligned}
\left\|v_{h}\right\|_{L^{\infty}(\partial E)}^{2} & \lesssim\left(h_{E}^{-1}\left\|v_{h}\right\|_{0, \partial E}^{2}+\left|v_{h}\right|_{1 / 2, \partial E}^{2}\right) \quad \forall v_{h} \in V_{E}, E \in \Omega_{h}, \\
|v|_{1 / 2, \partial E} & \lesssim|v|_{1, E} \quad \forall v \in H^{1}(E), E \in \Omega_{h}
\end{aligned}
$$

and

$$
h_{E}^{-1}\|v\|_{0, \partial E}^{2} \lesssim h_{E}^{-2}\|v\|_{0, E}^{2}+|v|_{1, E}^{2} \quad \forall v \in H^{1}(E), E \in \Omega_{h},
$$

proved in Lemmas 6.5 6.1 and 6.4 respectively. From 4.25) we thus obtain

$$
s_{E}^{\partial}\left(v_{h}, v_{h}\right) \lesssim h_{E}^{-2}\left\|v_{h}\right\|_{0, E}^{2}+\left|v_{h}\right|_{1, E}^{2} .
$$

Since $\Pi v_{h}=0$ implies $\mathcal{R} v_{h}=0$, bound (3.41) yields

$$
h_{E}^{-2}\left\|v_{h}\right\|_{0, E}^{2} \lesssim\left|v_{h}\right|_{1, E}^{2}
$$

The first bound in (4.23) now follows from combining (4.24), (4.29) and 4.30) and noting that $\left|v_{h}\right|_{1, E}^{2} \lesssim a_{E}\left(v_{h}, v_{h}\right)$. 


\subsection{A stabilization based on boundary derivatives}

We now analyze a different choice for the boundary part of the stabilization term, namely the one given by (cf. Ref. 34):

$$
s_{E}^{\partial}\left(v_{h}, w_{h}\right)=h_{E} \int_{\partial E} \partial_{s} v_{h} \partial_{s} w_{h} d s \quad \forall v_{h}, w_{h} \in V_{E} .
$$

We highlight that, contrary to the identity matrix stabilization presented in Sec.4.1 the standard approach of Ref. 4 applied to (4.31), would lead to a strongly suboptimal result in the presence of small edges. Indeed, the term 4.31) can be bounded by the $H^{1}$-semi-norm only with a constant $\alpha^{*} \simeq h_{E} / h_{m(E)}$ (cf. the second bound in Eq. (3.7) of Ref. 4). Instead, with the present analysis we can obtain uniform bounds only making use of assumption (A1). In fact, we have the following result.

Proposition 4.4. Let assumption (A1) hold. Then, for the boundary form (4.31), conditions (3.29) and (3.37) hold with positive constants $\widehat{C}_{1}$ and $\widehat{C}_{2}$ that satisfy:

$$
\widehat{C}_{1}(E) \lesssim 1, \quad \widehat{C}_{2}(E) \lesssim 1
$$

Proof. We first prove that condition (B.37) is fulfilled. Take any $p \in \mathbb{P}_{k}(E)$. Using assumption (A1) and an inverse inequality for polynomials (cf. Remark 6.1), we get

$$
\begin{aligned}
s_{E}^{\partial}((I-\mathcal{R}) p,(I-\mathcal{R}) p) & =h_{E}|(I-\mathcal{R}) p|_{1, \partial E}^{2} \lesssim h_{E}^{2}\|\nabla(I-\mathcal{R}) p\|_{L^{\infty}(\partial E)}^{2} \\
& \leq h_{E}^{2}\|\nabla(I-\mathcal{R}) p\|_{L^{\infty}(E)}^{2} \\
& \lesssim\|\nabla(I-\mathcal{R}) p\|_{0, E}^{2}=|p|_{1, E}^{2},
\end{aligned}
$$

i.e. condition (3.37) holds with $\widehat{C}_{2}(E) \lesssim 1$.

To prove that condition (3.29) is fulfilled, we simply notice that

$$
\begin{aligned}
\left|v_{h}\right|_{1 / 2, \partial E}^{2} \lesssim h_{E}\left|v_{h}\right|_{1, \partial E}^{2} & =h_{E}\left|(I-\mathcal{R}) v_{h}\right|_{1, \partial E}^{2} \\
& =s_{E}^{\partial}\left((I-\mathcal{R}) v_{h},(I-\mathcal{R}) v_{h}\right) \quad \forall v_{h} \in V_{E} .
\end{aligned}
$$

and we obtain that condition (3.29) holds with $\widehat{C}_{1}(E) \lesssim 1$.

Theorem 4.5. Let assumption (A1) hold. Let $u$ be the solution of problem (2.1), assumed to be in $H^{s}(\Omega), s>3 / 2$. Let $u_{h}$ be the solution of the discrete problem (2.13), with the choice 4.31). Then it holds

$$
\left\|u-u_{h}\right\|_{1, \Omega} \lesssim h^{s-1}|u|_{s, \Omega} \quad 3 / 2<s \leq k+1
$$

Proof. Proposition 4.4 allows to apply Propositions 3.6 and 3.7 Therefore, assumptions (3.2) and (3.3) hold with $C_{1}(E) \lesssim 1$ and $C_{2}(E) \lesssim 1$, respectively. Then, Theorem 3.2 can be invoked with $C_{\text {err }}(h)$ satisfying $C_{\text {err }}(h) \lesssim 1$. We now 
estimate the terms in the right-hand side of (3.16), choosing both $u_{\pi}$ and $u_{I}$ as in Theorem 4.2. Using exactly the same arguments of Theorem 4.2 we get:

$$
\begin{aligned}
\mathfrak{F}_{h} & \lesssim h^{k} \\
\left(\sum_{E \in \mathcal{T}_{h}}\left|\Pi_{E}\left(u-u_{I}\right)\right|_{1, E}^{2}\right)^{1 / 2} & \lesssim\left|u-u_{I}\right|_{1, \Omega} \lesssim h^{s-1}|u|_{s, \Omega} \quad 3 / 2<s \leq k+1 \\
\left(\sum_{E \in \mathcal{T}_{h}}\left|\Pi_{E}\left(u-u_{\pi}\right)\right|_{1, E}^{2}\right)^{1 / 2} & \lesssim\left(\sum_{E \in \mathcal{T}_{h}}\left|u-u_{\pi}\right|_{1, E}^{2}\right)^{1 / 2} \\
& \lesssim h^{s-1}|u|_{s, \Omega} \quad 3 / 2<s \leq k+1,
\end{aligned}
$$

and

$$
\begin{aligned}
& s_{E}^{\circ}\left((I-\mathcal{R})\left(u-u_{I}\right),(I-\mathcal{R})\left(u-u_{I}\right)\right) \\
& \quad \lesssim\left|u-u_{I}\right|_{1, E}^{2} \lesssim h_{E}^{2 s-2}|u|_{s, E}^{2} \quad 3 / 2<s \leq k+1 .
\end{aligned}
$$

Therefore, we only need to estimate the boundary part

$$
s_{E}^{\partial}\left((I-\mathcal{R})\left(u-u_{I}\right),(I-\mathcal{R})\left(u-u_{I}\right)\right)=s_{E}^{\partial}\left(u-u_{I}, u-u_{I}\right) .
$$

To this end, take $s>3 / 2$ and $\sigma$ such that $3 / 2<\sigma<s$. We have, using a scaled trace inequality (proved by a similar argument to that in Lemma 6.1 for the function $\left.\nabla\left(u-u_{I}\right)\right)$ :

$$
s_{E}^{\partial}\left(u-u_{I}, u-u_{I}\right)=h_{E}\left|u-u_{I}\right|_{1, \partial E}^{2} \lesssim\left|u-u_{I}\right|_{1, E}^{2}+h_{E}^{2 \sigma-2}\left|u-u_{I}\right|_{\sigma, E}^{2} .
$$

Hence, Theorem 3.4 gives:

$$
s_{E}^{\partial}\left(u-u_{I}, u-u_{I}\right) \lesssim\left|u-u_{I}\right|_{1, E}^{2}+h_{E}^{2 \sigma-2}\left|u-u_{I}\right|_{\sigma, E}^{2} \lesssim h_{E}^{2 s-2}|u|_{s, E}^{2} .
$$

Combining (4.38) and 4.40), we get

$$
\left\|u-u_{I}\right\| \lesssim\left(\sum_{E \in \mathcal{T}_{h}} h_{E}^{2 s-2}|u|_{s, E}^{2}\right)^{1 / 2} \lesssim h^{s-1}|u|_{s, \Omega} .
$$

Similarly, using also standard approximation results on polygons (see Ref. 22), we get

$$
\left\|u-u_{\pi}\right\| \lesssim\left(\sum_{E \in \mathcal{T}_{h}} h_{E}^{2 s-2}|u|_{s, E}^{2}\right)^{1 / 2} \lesssim h^{s-1}|u|_{s, \Omega} .
$$

We conclude by collecting estimates 4.35), (4.36), 4.37), 4.41) and (4.42).

Remark 4.1. The same analysis can be employed to prove error estimates for many other choices of the stabilization. We here consider the following variants of choice 4.31). 
The first variant is an " $L^{2}$-version" of (4.31):

$$
s_{E}^{\partial}\left(v_{h}, w_{h}\right)=\sum_{e \in \partial E} h_{e}^{-1} \int_{e} v_{h} w_{h} d s \quad \forall v_{h}, w_{h} \in V_{E} .
$$

It is easy to check that, under assumptions (A1) and (A2), it holds

$$
\sum_{e \in \partial E} h_{e}^{-1}\left\|v_{h}\right\|_{0, E}^{2} \simeq\left\|v_{h}\right\|_{L^{\infty}}^{2} \quad \forall v_{h} \in V_{h \mid E} .
$$

Hence, by following the same steps used for the identity matrix choice of Sec. 4.1 one can easily obtain that Proposition 4.1 holds also for the present choice.

A second possible choice would be to substitute $h_{e}^{-1}$ with $h_{E}^{-1}$ in (4.43). For this choice, robust results (at least from the theoretical perspective of the present analysis) would be obtained only under the stronger assumption (A3). Indeed, it is easy to check that for this latter choice one would get a factor $h_{E} / h_{m(E)}$ in the constant of bound (3.29).

\section{Numerical Tests}

For all numerical tests we will consider Laplace equation on the unit square $\Omega:=$ ] $0,1[2$ :

$$
\begin{cases}-\Delta u=f & \text { in } \Omega \\ u=g & \text { on } \partial \Omega\end{cases}
$$

with right-hand side $f$ and Dirichlet boundary condition $g$ defined in such a way that the exact solution is (see Fig. 11):

$$
\begin{aligned}
u_{\mathrm{ex}}(x, y):= & x^{3}-x y^{2}+x^{2} y+x^{2}-x y-x \\
& +y-1+\sin (5 x) \sin (7 y)+\log \left(1+x^{2}+y^{4}\right) .
\end{aligned}
$$

In the following brief experiments we will address some of the issues considered in the paper.

Remark 5.1. First of all, we point out that in all our experiments we have observed a very weak dependence of the VEM solution with respect to the inclusion in the stabilization of the term $s_{E}^{\circ}\left(v_{h}, w_{h}\right)$ depending on the internal degrees of freedom. This observation holds for all kinds of boundary stabilization adopted. Hence, we have set everywhere $s_{E}^{\circ}\left(v_{h}, w_{h}\right)=0$ (see Sec. 3.1).

\subsection{Small edges}

In the first numerical experiment we consider the issue of the presence of very small edges. On the one hand, we show that the classical VEM stabilization (4.1) can generate small oscillations, that are of the order of the approximation error. In the case $k=1$ these oscillations are visible and, depending on the application, may be preferable to avoid. However, already for $k=2$ the oscillations become so 


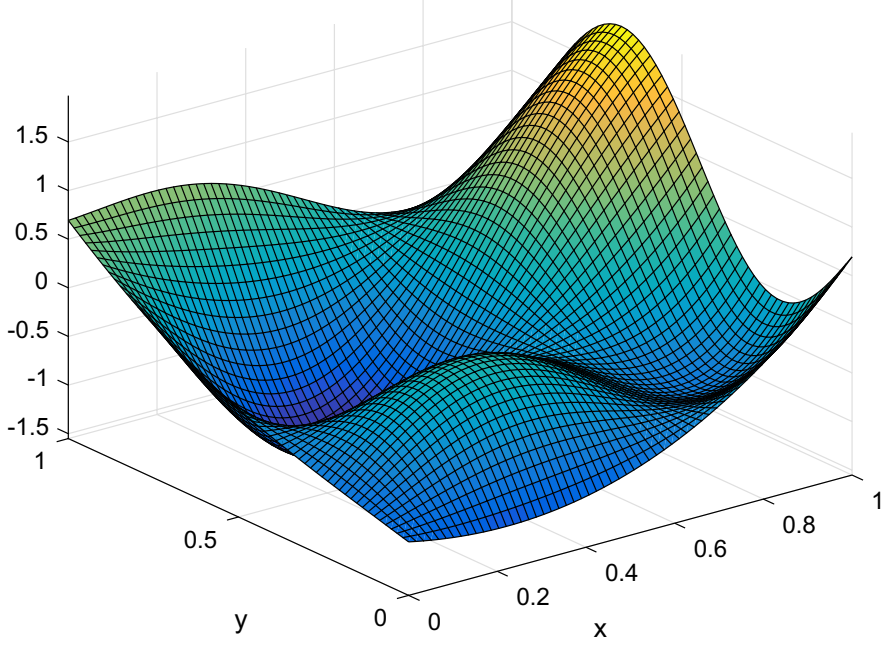

Fig. 1. Exact solution.

small to be practically negligible. On the other hand, we show that the stabilization (4.31) eliminates this oscillations already for the $k=1$ case.

We consider a mesh obtained by gluing together two distinct meshes along $x=0.5$; this case can happen for instance in contact problems, see Ref. 34. The mesh is shown in Fig. 2, while in Fig. 3 we show the section of the mesh at $x=0.5$. Note that around $y=0.4$ there is a very small edge of length $3.21 \times 10^{-4}$.

In Figs. 4 and 5 we plot the section at $x=0.5$ of the VEM solution for the classical stabilization (4.1) (thick line) together with the exact solution (thinner line) for $k=1$ and $k=2$, respectively. A careful inspection shows that in Fig. 4 there are

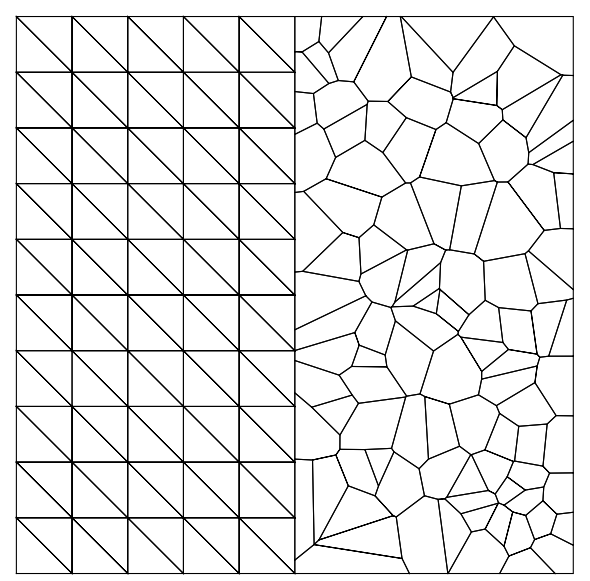

Fig. 2. Mesh. 


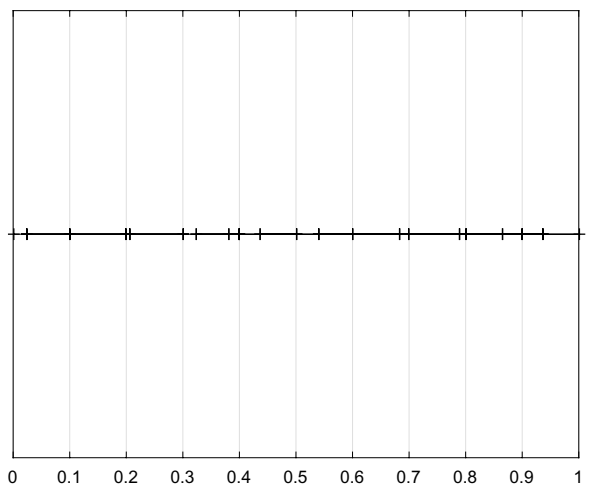

Fig. 3. Mesh section at $x=0.5$.

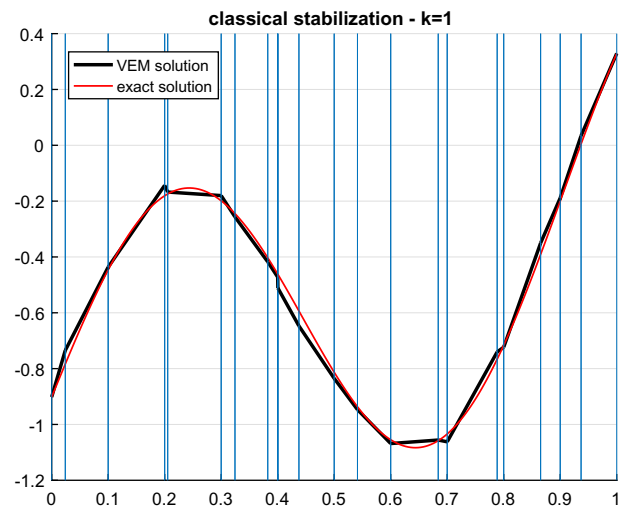

Fig. 4. Exact solution and VEM solution for $k=1$ and classical stabilization (4.1).

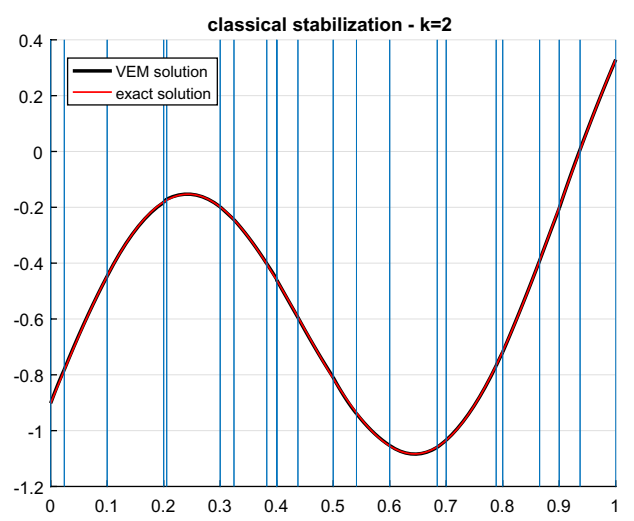

Fig. 5. Exact solution and VEM solution for $k=2$ and classical stabilization (4.1). 


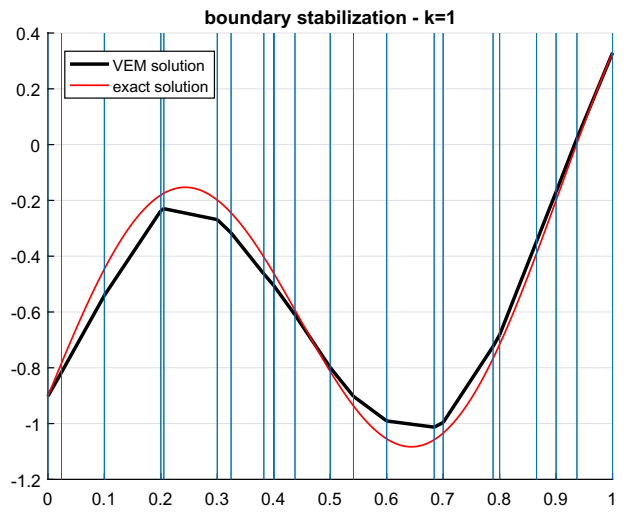

Fig. 6. Exact solution and VEM solution for $k=1$ and boundary stabilization 4.31).

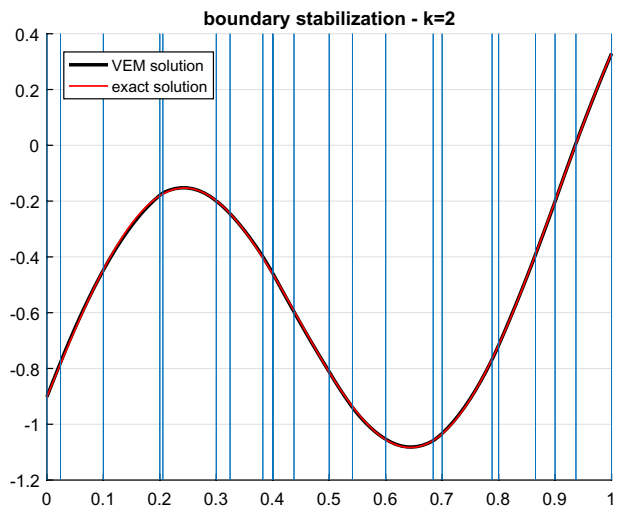

Fig. 7. Exact solution and VEM solution for $k=2$ and boundary stabilization 4.31).

some small oscillations in correspondence of the small edges. In Fig. [5, the oscillations are no more visible but are still present. We reproduce the same experiments in Figs. 6 and 7 with the boundary stabilization (4.31). Now the oscillation have disappeared also for $k=1$ but in this case the VEM solution seems to be less accurate. The motivation is that the boundary stabilization (4.31) is too strong. The situation can be improved by taking a smaller stabilization parameter (see Remark 2.1); in Figs. 8 and 9 we show the same experiments with $\tau_{E}=\tau=0.1$. We have developed several further experiments (here not shown) using different meshes and loading, and choosing $\tau=0.1$ for $s_{E}(\cdot, \cdot)$ as in 4.31): the obtained results were always accurate. This is a general property of VEM: the sensitivity of the method with respect to the stabilization parameter is very mild when considering different meshes and loading/boundary data. Nevertheless, a detailed study on such an issue is beyond the scope of this paper. 


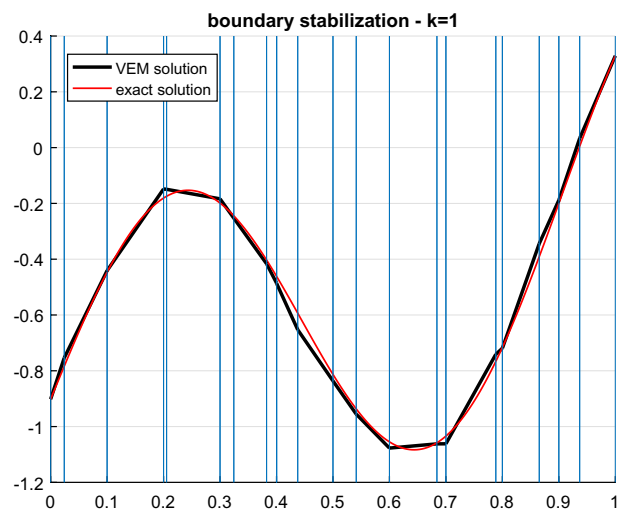

Fig. 8. Exact solution and VEM solution for $k=1$, boundary stabilization 4.31 and $\tau=0.1$.

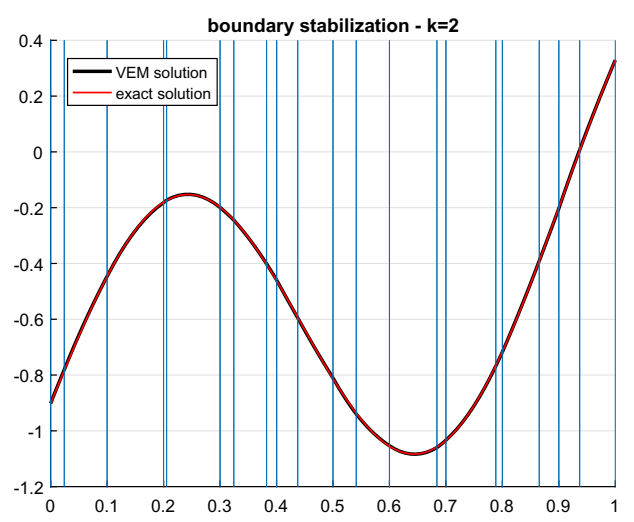

Fig. 9. Exact solution and VEM solution for $k=2$, boundary stabilization 4.31 and $\tau=0.1$.

\subsection{Convergence in $H^{1}$}

We will show, in a log-log scale, the convergence curves of the error in the $H^{1}$-seminorm between the exact solution $u_{\mathrm{ex}}$ and the solution $u_{h}$ given by the VEM. As the VEM solution $u_{h}$ is not explicitly known inside the elements, we compare $\nabla u_{\mathrm{ex}}$ with the elementwise $L^{2}$-projection of $\nabla u_{h}$ onto $\mathbb{P}_{k-1}$, that is, with $\Pi_{k-1}^{0} \nabla u_{h}$. It is easy to see that this latter quantity can indeed be computed starting from the degrees of freedom of $u_{h}$. For the convergence test we consider four sequences of meshes.

The first sequence of meshes (labeled square) is simply a decomposition of the domain in $4 \times 4,8 \times 8,16 \times 16$ and $32 \times 32$ equal squares, and the second one (labeled hexagon) is a decomposition of the domain in $8 \times 10,18 \times 20,26 \times 30,34 \times 40$ and $44 \times 50$ (almost) regular hexagons. The first meshes of the two sequences are shown in Fig. 10 and in Fig. 111, respectively. 


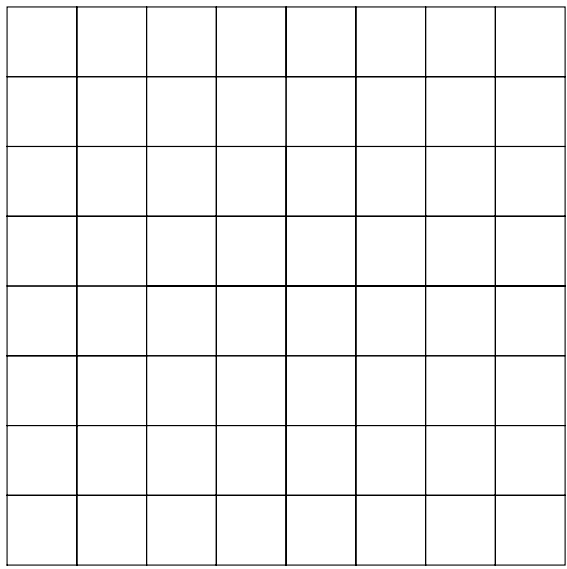

Fig. 10. Square mesh.

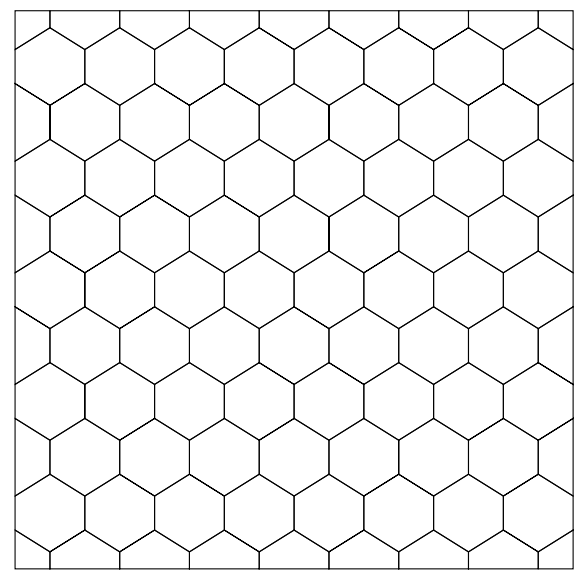

Fig. 11. Hexagon mesh.

The third sequence of meshes (labeled Lloyd-0) is a random Voronoi polygonal tessellation of the unit square in 25, 100, 400 and 1600 polygons. The fourth sequence (labeled Lloyd-100) is obtained starting from the previous one and performing 100 Lloyd iterations leading to a Centroidal Voronoi Tessellation (CVT) (see e.g. Ref.21). The 100-polygon mesh of each family is shown in Fig.12(Lloyd-0) and in Fig. 13 (Lloyd-100), respectively.

From Figs. 14 17 we plot for $k=1$ (low-order case) the $H^{1}$-error on each mesh family as a function of the mean diameter $h$ of the polygons. We consider the classical stabilization (4.1) (solid line), the boundary stabilization (4.31) with $\tau=1$ (dotted line), and the boundary stabilization (4.31) with $\tau=0.1$ (dashed 


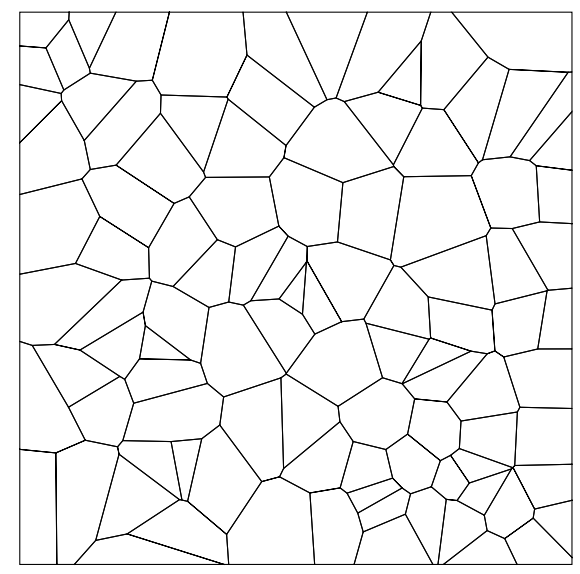

Fig. 12. Lloyd-0 mesh.

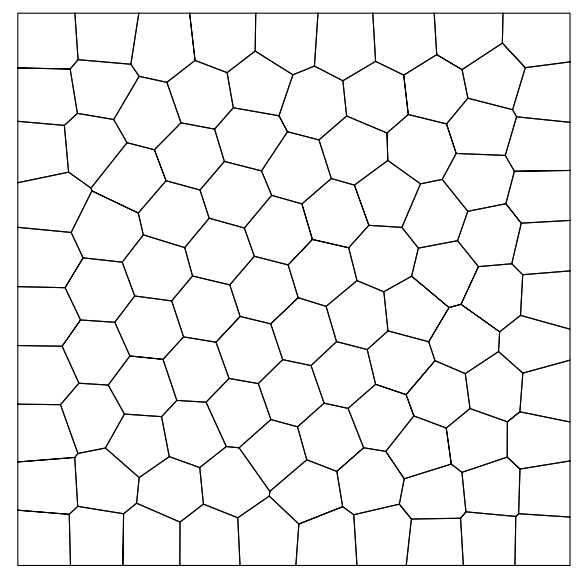

Fig. 13. Lloyd-100 mesh.

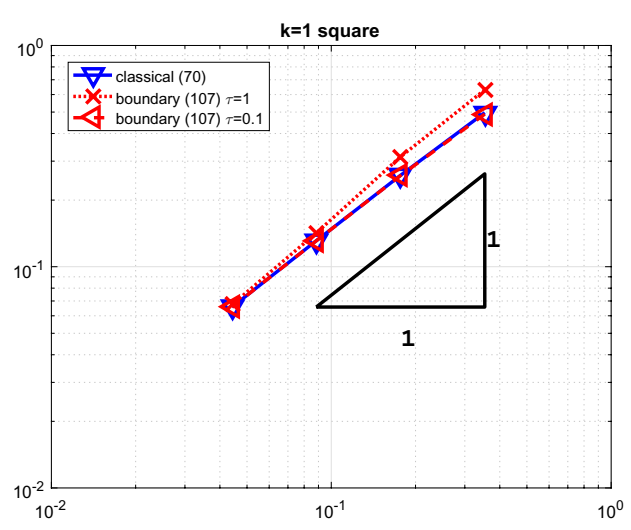

Fig. 14. $\quad k=1$, Square mesh. 


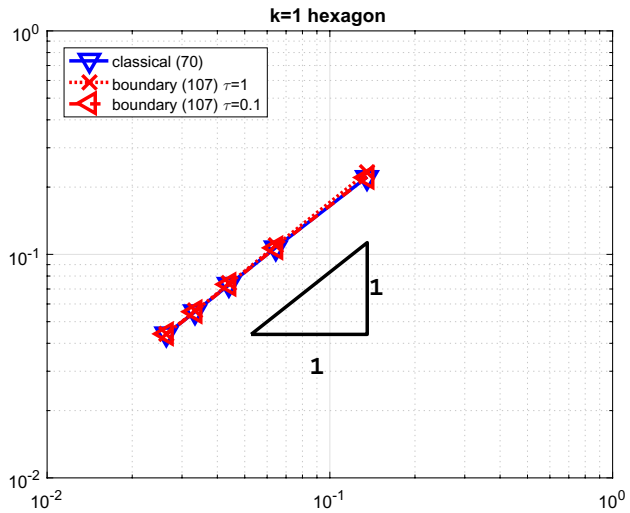

Fig. 15. $k=1$, Hexagon mesh.

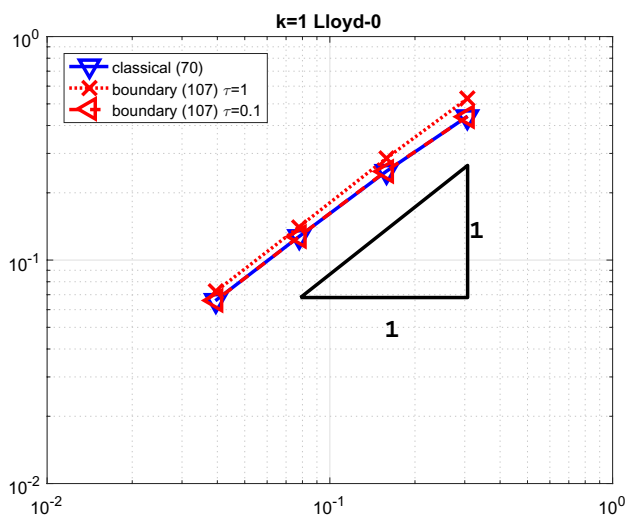

Fig. 16. $k=1$, Lloyd-0 mesh.

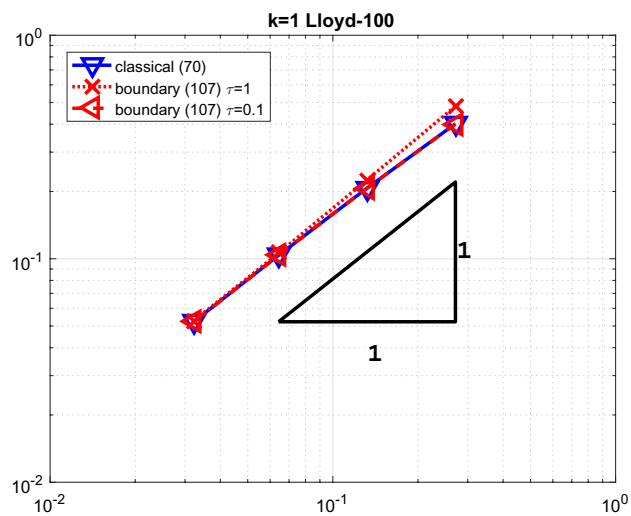

Fig. 17. $k=1$, Lloyd-100 mesh. 


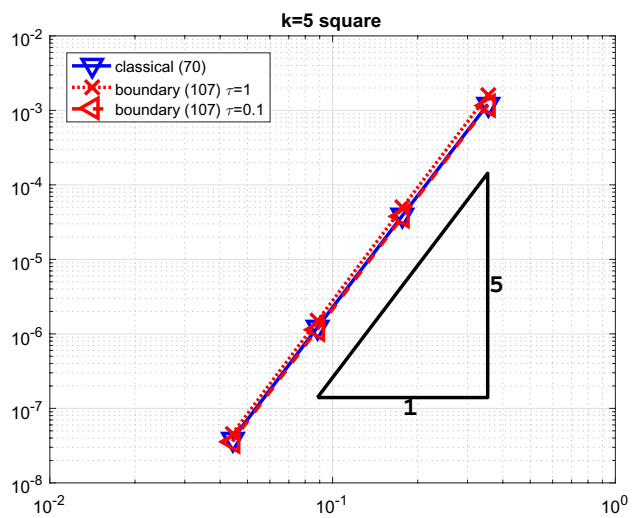

Fig. 18. $k=5$, Square mesh.

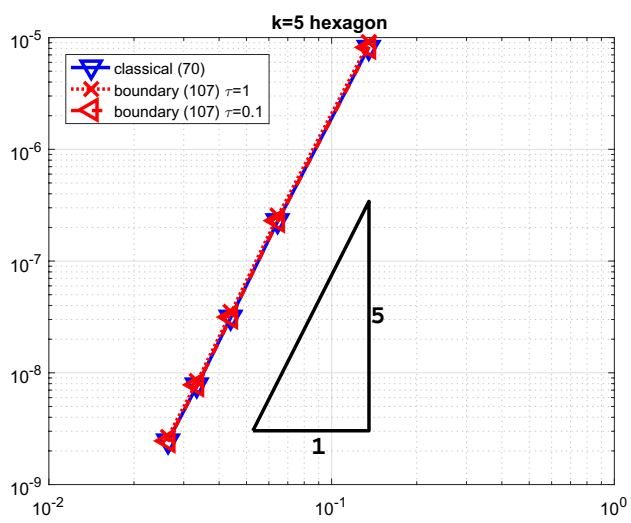

Fig. 19. $k=5$, Hexagon mesh.

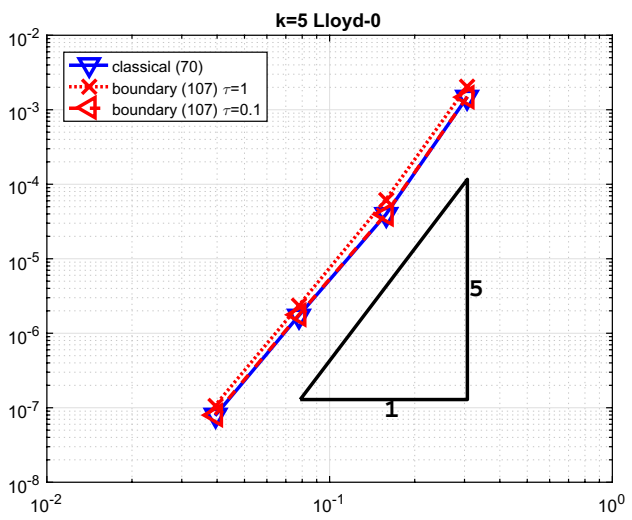

Fig. 20. $k=5$, Lloyd-0 mesh. 


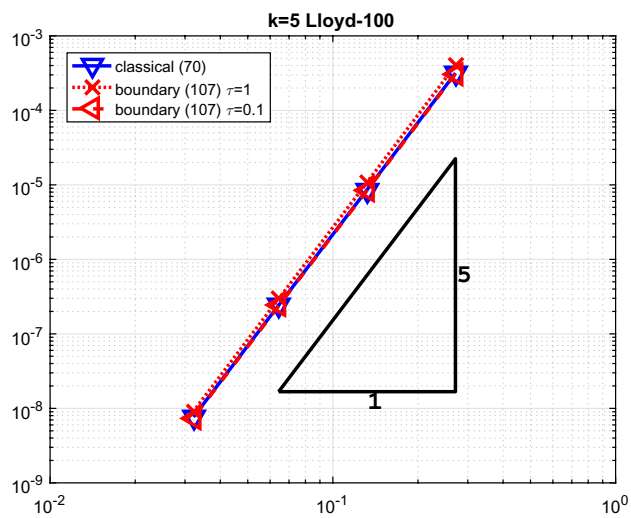

Fig. 21. $k=5$, Lloyd-100 mesh.

line). From Figs. 18 21, we finally plot the same values for $k=5$ (as a sample high-order case).

We observe that as $h$ goes to zero all stabilizations behave very similarly, namely as $O\left(h^{k}\right)$, as predicted by the theory.

\section{Technical Tools}

In this section, we collect a number of technical results that are used in the paper. We remark that some similar estimates have been independently proved in Ref. 14.

We begin with the following lemmas.

Lemma 6.1. Let assumption (A1) hold. Then

$$
|v|_{1 / 2, \partial E} \lesssim|v|_{1, E} \quad \forall v \in H^{1}(E), E \in \Omega_{h} .
$$

Moreover, for all $E \in \Omega_{h}$ and all $v \in H^{1 / 2}(\partial E)$, there exists an extension $\widetilde{v} \in$ $H^{1}(E)$ such that

$$
|\widetilde{v}|_{1, E} \lesssim|v|_{1 / 2, \partial E}
$$

Proof. We only sketch the simple proof, based on a mapping argument. Up to a translation of the element $E$, we may assume that the ball center $\mathbf{x}_{E}$ is the origin of the coordinate axes. Let then the function $\Psi:[0,2 \pi) \rightarrow\left[\rho_{E}, h_{E}\right]$ describe the boundary of $E$, as follows. The boundary curve $\Gamma=\partial E$ can be parametrized in a unique way as

$$
\gamma(\theta)=(\Psi(\theta) \cos (\theta), \Psi(\theta) \sin (\theta)), \quad \theta \in[0,2 \pi),
$$

with $\theta$ representing the angle in radial coordinates. Note that property (A1) implies $\Psi \in W^{1, \infty}[0,2 \pi)$, uniformly with respect to $E \in \Omega_{h}$. We then introduce the radial 
mapping $F: \bar{B}_{E} \rightarrow \bar{E}$, associating a point expressed in polar coordinates

$$
(\hat{x}, \hat{y})=(\hat{r} \cos (\hat{\theta}), \hat{r} \sin (\hat{\theta})), \quad \hat{r} \in\left[0, \rho_{E}\right], \hat{\theta} \in[0,2 \pi),
$$

with the point $(x, y)=F(\hat{x}, \hat{y})$, whose coordinates are

$$
(x, y)=(r \cos (\theta), r \sin (\theta)), \quad r=\hat{r} \frac{\Psi(\hat{\theta})}{\rho_{E}}, \theta=\hat{\theta} .
$$

By recalling (A1), it can be checked that $F \in W^{1, \infty}\left(B_{E}\right)$, and the same holds for the inverse mapping, i.e. $F^{-1} \in W^{1, \infty}(E)$. It is easy to see that $|F|_{W^{1, \infty}\left(B_{E}\right)} \lesssim C$ and $\left|F^{-1}\right|_{W^{1, \infty}(E)} \lesssim C$. As a consequence, bound (6.1) can be simply proved by a standard "pull-back and push-forward" argument: (i) map $v \in H^{1}(E)$ from $E$ into $B_{E}$ using $F$; (ii) notice that the trace bound analogous to (6.1) holds on the ball $B_{E}$; (iii) map back to $E$ using $F^{-1}$. Bound (6.2) is similarly proved: one only needs to map the boundary data into $B_{E}$, to consider the harmonic extension inside $B_{E}$, and finally to map back to $E$.

Lemma 6.2. Let assumption (A1) hold. Then

$$
\left|\mathbf{w} \cdot \mathbf{n}_{E}\right|_{H^{-1 / 2}(\partial E)} \lesssim\|\mathbf{w}\|_{0, E} \quad \forall \mathbf{w} \in\left[L^{2}(E)\right]^{2} \quad \text { with div } \mathbf{w}=0, \quad \forall E \in \Omega_{h},
$$

with $\mathbf{n}_{E}$ denoting the outward unit normal to the boundary of $E$.

Proof. By the definition of dual norm and using 6.2, we get

$$
\begin{aligned}
&\left|\mathbf{w} \cdot \mathbf{n}_{E}\right|_{H^{-1 / 2}(\partial E)}=\sup _{v \in H^{1 / 2}(\partial E)} \frac{-1 / 2, \partial E}{\left|\mathbf{w} \cdot \mathbf{n}_{E}, v\right\rangle_{1 / 2, \partial E}} \\
& \lesssim \sup _{\widetilde{v} \in H^{1}(E)} \frac{-1 / 2, \partial E}{\left.|v| \mathbf{w} \cdot \mathbf{n}_{E}, \widetilde{v}\right\rangle_{1 / 2, \partial E}} \\
&|\widetilde{v}|_{1, E}
\end{aligned}
$$

An integration by parts, using $\operatorname{div} \mathbf{w}=0$, and the Cauchy-Schwarz inequality lead to estimate (6.4):

$$
\left|\mathbf{w} \cdot \mathbf{n}_{E}\right|_{H^{-1 / 2}(\partial E)} \lesssim \sup _{\widetilde{v} \in H^{1}(E)} \frac{\int_{E} \mathbf{w} \cdot \nabla \widetilde{v}}{|\widetilde{v}|_{1, E}} \leq\|\mathbf{w}\|_{0, E} .
$$

Lemma 6.3. Let assumption (A1) hold true. Then we have:

$$
\left\|\Delta v_{h}\right\|_{0, E} \lesssim h_{E}^{-1}\left|v_{h}\right|_{1, E} \quad \forall v_{h} \in V_{E} .
$$

Proof. For $E \in \Omega_{h}$, let $T_{E} \subset E$ denote an equilateral triangle inscribed in the ball $B_{E}$. We start observing that, due to assumption (A1), for any polynomial $p$ of given maximum degree it holds $\|p\|_{0, E} \lesssim\|p\|_{0, T_{E}}$. This follows from noting that the smallest ball containing $E$ and the largest ball contained in $T_{E}$ have uniformly comparable radii. We now recall that $\Delta v_{h} \in \mathbb{P}_{k-2}$. Let $b \in \mathbb{P}_{3}\left(T_{E}\right)$ denote the 
standard cubic bubble in $T_{E}$ with unitary maximum value. Standard properties and inverse estimates of polynomial spaces on shape regular triangles yield

$$
\begin{aligned}
\left\|\Delta v_{h}\right\|_{0, E}^{2} & \lesssim\left\|\Delta v_{h}\right\|_{0, T_{E}}^{2} \lesssim \int_{T_{E}} b \Delta v_{h} \Delta v_{h}=\int_{T_{E}} \nabla v_{h} \cdot \nabla\left(b \Delta v_{h}\right) \\
& \lesssim\left|v_{h}\right|_{1, T_{E}} h_{E}^{-1}\left\|b \Delta v_{h}\right\|_{0, T_{E}} \leq h_{E}^{-1}\left|v_{h}\right|_{1, T_{E}}\left\|\Delta v_{h}\right\|_{0, T_{E}} .
\end{aligned}
$$

Estimate (6.5) now follows by observing $\left\|\Delta v_{h}\right\|_{0, T_{E}} \leq\left\|\Delta v_{h}\right\|_{0, E}$.

Remark 6.1. The same argument in the proof of Lemma 6.3 can be used to prove inverse estimates for polynomials of fixed maximum degree, on polygons satisfying assumption (A1).

Lemma 6.4. Let assumption (A1) hold. We have

$$
h_{E}^{-1}\|v\|_{0, \partial E}^{2} \lesssim h_{E}^{-2}\|v\|_{0, E}^{2}+|v|_{1, E}^{2} \quad \forall E \in \Omega_{h},
$$

and for all $v$ in $H^{1}(E)$.

Proof. The simple proof is based on an anisotropic scaling argument. Take an edge $e \in \partial E$, and let $T \in \mathcal{T}_{h}$ be the associated triangle (see the proof of Theorem 3.4). By a rotation and translation of the Cartesian $(x, y)$-coordinates, it is not restrictive to assume that $e=\{0\} \times\left[-h_{e} / 2, h_{e} / 2\right]$, and that the center of the ball, see assumption (A1), $\mathbf{x}_{E}=\left(x_{E}, y_{E}\right)$ satisfies $x_{E} \geq 0$. As a consequence of assumption (A1), it is easy to check that $x_{E} \lesssim h_{E}, y_{E} \lesssim h_{E}$ and that the ball $B_{E}$ is contained in the half-plane $\left\{(x, y) \in \mathbb{R}^{2}: x \geq 0\right\}$. Therefore, we also have $x_{E} \gtrsim h_{E}$. Let $\hat{T}$ now be the triangle of vertices $\left(0, h_{e} / 2\right),\left(0,-h_{e} / 2\right),\left(h_{e} / 2,0\right)$. We now consider the unique affine mapping $F: T \rightarrow \hat{T}$ that leaves the edge $e$ (and its orientation) unchanged: $\hat{e}:=F(e)=e$. By an explicit computation of $F$ and its inverse $F^{-1}$, we get the Jacobian matrices:

$$
D F=\left(\begin{array}{cc}
h_{e} / x_{E} & 0 \\
-y_{E} / x_{E} & 1
\end{array}\right), \quad D F^{-1}=\left(\begin{array}{ll}
x_{E} / h_{e} & 0 \\
y_{E} / h_{e} & 1
\end{array}\right) .
$$

The proof now follows by a scaling argument. Indeed, denoting $\hat{v}=v \circ F^{-1}$, wellknown (scaled) trace estimates on $\hat{T}$ and a simple change of variables give

$$
\begin{aligned}
\|v\|_{0, e}^{2} & =\|\hat{v}\|_{0, \hat{e}}^{2} \lesssim h_{e}^{-1}\|\hat{v}\|_{0, \hat{T}}^{2}+h_{e}|\hat{v}|_{1, \hat{T}}^{2} \\
& \lesssim h_{e}^{-1} \frac{h_{e}}{x_{E}}\|v\|_{0, T}^{2}+h_{e} \frac{h_{e}}{x_{E}}\left(\left(\frac{x_{E}}{h_{e}}\right)^{2}+\left(\frac{y_{E}}{h_{e}}\right)^{2}\right)\left\|\frac{\partial v}{\partial x}\right\|_{0, T}^{2}+h_{e} \frac{h_{e}}{x_{E}}\left\|\frac{\partial v}{\partial y}\right\|_{0, T}^{2} .
\end{aligned}
$$

By recalling the upper and lower bounds on $\left(x_{E}, y_{E}\right)$, the above estimate yields

$$
\|v\|_{0, e}^{2} \lesssim h_{E}^{-1}\|v\|_{0, T}^{2}+h_{E}\|\nabla v\|_{0, T}^{2},
$$

that immediately implies (6.6) by summing overall $e \in \partial E$. 
The next lemma can be considered as a variant of Lemma 3.1 in Ref. 13, supposing that the number of edges is uniformly bounded.

Lemma 6.5. Let (A1) and (A2) hold. For all $E \in \Omega_{h}$ and all $v_{h} \in V_{E}$ we have

$$
\left\|v_{h}\right\|_{L^{\infty}(\partial E)}^{2} \lesssim\left(h_{E}^{-1}\left\|v_{h}\right\|_{0, \partial E}^{2}+\left|v_{h}\right|_{1 / 2, \partial E}^{2}\right)
$$

Proof. For $w_{h} \in V_{E}$, we recall that $w_{h \mid \partial E} \in C^{0}(\partial E)$ and $w_{h \mid \partial E}$ is a polynomial of degree at most $k$ on each edge.

In addition, we first suppose that $\int_{\partial E} w_{h}=0$. Then, by definition (2.14) and by using a scaling argument on each edge of the mesh, we obtain

$$
\left|w_{h}\right|_{1 / 2, \partial E}^{2} \geq \sum_{e \in \partial E}\left|w_{h}\right|_{1 / 2, e}^{2} \gtrsim \sum_{e \in \partial E}\left\|\frac{\partial w_{h}}{\partial s}\right\|_{L^{1}(e)}^{2}
$$

where $s$ denotes the curvilinear abscissae along the generic edge. By assumption (A2) and recalling that $w_{h}$ is continuous on the boundary, from the above bound we have:

$$
\left|w_{h}\right|_{1 / 2, \partial E}^{2} \gtrsim\left(\sum_{e \in \partial E}\left\|\frac{\partial w_{h}}{\partial s}\right\|_{L^{1}(e)}\right)^{2}=\left\|\frac{\partial w_{h}}{\partial s}\right\|_{L^{1}(\partial E)}^{2} \geq\left\|w_{h}\right\|_{L^{\infty}(\partial E)}^{2},
$$

where we also used that $\left.w_{h}\right|_{\partial E}$ has zero average and thus it vanishes at least at one point of $\partial E$. For a generic $v_{h} \in V_{E}$ (not necessarily with vanishing mean value), the proof follows easily from (6.7) by adding and subtracting its average on the boundary $\bar{v}_{h}$ and simple bounds:

$$
\begin{aligned}
\left\|v_{h}\right\|_{L^{\infty}(\partial E)} & \leq\left\|v_{h}-\bar{v}_{h}\right\|_{L^{\infty}(\partial E)}+\left\|\bar{v}_{h}\right\|_{L^{\infty}(\partial E)} \\
& \lesssim\left|v_{h}-\bar{v}_{h}\right|_{1 / 2, \partial E}+|\partial E|^{-1 / 2}\left\|\bar{v}_{h}\right\|_{0, \partial E} \\
& \lesssim\left|v_{h}\right|_{1 / 2, \partial E}+|\partial E|^{-1 / 2}\left\|v_{h}\right\|_{0, \partial E} \\
& \lesssim\left|v_{h}\right|_{1 / 2, \partial E}+h_{E}^{-1 / 2}\left\|v_{h}\right\|_{0, \partial E},
\end{aligned}
$$

where $|\partial E|$ denotes the length of $\partial E$.

Lemma 6.6. Let assumptions (A1) and (A2) hold. For all $E \in \Omega_{h}$ and all $v_{h} \in V_{E}$ we have

$$
\left|v_{h}\right|_{1 / 2, \partial E}^{2} \lesssim \widehat{C}(E)\left\|v_{h}\right\|_{L^{\infty}(\partial E)}^{2}
$$

with $\widehat{C}(E)=\left(\log \left(1+h_{E} / h_{m(E)}\right)\right)$.

Proof. We first recall that $\partial E$ is meshed by means of its edges, so that $\partial E=$ $\bigcup_{j=1}^{N} e_{j}$. We also define $h_{j}:=\left|e_{j}\right|$. Moreover, in the proof we will make use of the 
space $H_{00}^{1 / 2}(\Gamma)$, where $\Gamma$ is a connected part of $\partial E$ with $|\Gamma|>0$. This space is defined by, see Ref. 26.

$$
H_{00}^{1 / 2}(\Gamma)=\left\{v \in H^{1 / 2}(\Gamma): \operatorname{Ext}(v) \in H^{1 / 2}(\partial E)\right\},
$$

where $\operatorname{Ext}(v)$ denotes the extension by zero of $v$ to the whole $\partial E$. Its norm is defined by

$$
\|v\|_{H_{00}^{1 / 2}(\Gamma)}:=\left(|v|_{1 / 2, \Gamma}^{2}+\int_{\Gamma} \frac{v(x)^{2}}{\rho(x)} d x\right)^{1 / 2},
$$

where $\rho(x)$ denotes the distance of $x$ from $\partial \Gamma$, is equivalent to $|\operatorname{Ext}(v)|_{1 / 2, \partial E}$.

Given $v_{h} \in V_{E}$, we set $v_{L} \in V_{E}$ as the usual piecewise linear Lagrange interpolant of $v_{h}$, relative to the edge mesh. We have

$$
\left|v_{h}\right|_{1 / 2, \partial E}^{2} \lesssim\left|v_{h}-v_{L}\right|_{1 / 2, \partial E}^{2}+\left|v_{L}\right|_{1 / 2, \partial E}^{2} .
$$

We now define $w_{j}=\chi_{e_{j}}\left(v_{h}-v_{L}\right)$ and we notice that, since $v_{h}-v_{L}$ vanishes at all the nodes, we have

$$
\begin{aligned}
\left|v_{h}-v_{L}\right|_{1 / 2, \partial E} & =\left|\sum_{j=1}^{N} \operatorname{Ext}\left(w_{j}\right)\right|_{1 / 2, \partial E} \\
& \leq \sum_{j=1}^{N}\left|\operatorname{Ext}\left(w_{j}\right)\right|_{1 / 2, \partial E} \lesssim \sum_{j=1}^{N}\left\|w_{j}\right\|_{H_{00}^{1 / 2}\left(e_{j}\right)} .
\end{aligned}
$$

Exploiting that $w_{j}$ is a polynomial of degrees $\leq k$ on $e_{j}$, a scaling argument shows that

$$
\left\|w_{j}\right\|_{H_{00}^{1 / 2}\left(e_{j}\right)} \lesssim\left\|w_{j}\right\|_{L^{\infty}\left(e_{j}\right)}
$$

Therefore, recalling assumption (A2) and using that $\left\|v_{L}\right\|_{L^{\infty}(\partial E)} \lesssim\left\|v_{h}\right\|_{L^{\infty}(\partial E)}$, it holds

$$
\left|v_{h}-v_{L}\right|_{1 / 2, \partial E} \lesssim \sum_{j=1}^{N}\left\|w_{j}\right\|_{L^{\infty}\left(e_{j}\right)} \lesssim\left\|v_{h}-v_{L}\right\|_{L^{\infty}(\partial E)} \lesssim\left\|v_{h}\right\|_{L^{\infty}(\partial E)},
$$

by which

$$
\left|v_{h}-v_{L}\right|_{1 / 2, \partial E}^{2} \lesssim\left\|v_{h}\right\|_{L^{\infty}(\partial E)}^{2} .
$$

It remains to estimate $\left|v_{L}\right|_{1 / 2, \partial E}^{2}$. We denote by $\varphi_{i}$ the usual hat function with support $\sigma_{i}:=e_{i-1} \cup e_{i}$ (here $i-1$ and $i$ are intended modulo $N$ ). We write

$$
v_{L}=\sum_{i=1}^{N} v_{i} \varphi_{i}
$$

where $v_{i} \in \mathbb{R}$ is the value of $v_{L}$ at the $i$ th node. We have, using assumption (A2):

$$
\left|v_{L}\right|_{1 / 2, \partial E}^{2} \lesssim\left\|v_{L}\right\|_{L^{\infty}(\partial E)}^{2} \sum_{i=1}^{N}\left|\varphi_{i}\right|_{1 / 2, \partial E}^{2} \lesssim\left\|v_{L}\right\|_{L^{\infty}(\partial E)}^{2} \sum_{i=1}^{N}\left\|\varphi_{i}\right\|_{H_{00}^{1 / 2}\left(\sigma_{i}\right)}^{2} .
$$


Recalling (6.10), direct computations show that

$$
\left|\varphi_{i}\right|_{H^{1 / 2}\left(\sigma_{i}\right)}^{2} \lesssim 1 ; \quad \int_{\sigma_{i}} \frac{\varphi_{i}(x)^{2}}{\rho(x)} d x \lesssim \log \left(1+\frac{\max \left\{h_{i-1}, h_{i}\right\}}{\min \left\{h_{i-1}, h_{i}\right\}}\right),
$$

by which we obtain

$$
\left\|\varphi_{i}\right\|_{H_{00}^{1 / 2}\left(\sigma_{i}\right)}^{2} \lesssim \log \left(1+\frac{\max \left\{h_{i-1}, h_{i}\right\}}{\min \left\{h_{i-1}, h_{i}\right\}}\right) .
$$

Therefore, using again assumption (A2) and noting that

$$
\frac{\max \left\{h_{i-1}, h_{i}\right\}}{\min \left\{h_{i-1}, h_{i}\right\}} \leq \frac{h_{E}}{h_{m(E)}} \quad 1 \leq i \leq N
$$

from (6.15) and 6.16) we get

$$
\left|v_{L}\right|_{1 / 2, \partial E}^{2} \lesssim \log \left(1+\frac{h_{E}}{h_{m(E)}}\right)\left\|v_{L}\right\|_{L^{\infty}(\partial E)}^{2} .
$$

Combining 6.11), 66.14 and (6.18), we get 6.8).

\section{Acknowledgments}

The first author was partially supported by the European Research Council through the H2020 Consolidator Grant (Grant No. 681162) CAVE - Challenges and Advancements in Virtual Elements. This support is gratefully acknowledged. The third author was partially supported by the research funds of the University of Milano-Bicocca. This support is gratefully acknowledged. All the authors were partially supported by IMATI-CNR. This support is gratefully acknowledged.

\section{References}

1. B. Ahmad, A. Alsaedi, F. Brezzi, L. D. Marini and A. Russo, Equivalent projectors for virtual element methods, Comput. Math. Appl. 66 (2013) 376-391.

2. T. Apel, Anisotropic Finite Elements: Local Estimates and Applications, Advances in Numerical Mathematics (Teubner, 1999).

3. C. Baiocchi and A. Capelo, Variational and Quasivariational Inequalities: Applications to Free Boundary Problems (Wiley, 1984).

4. L. Beirão da Veiga, F. Brezzi, A. Cangiani, G. Manzini, L. D. Marini and A. Russo, Basic principles of virtual element methods, Math. Models Methods Appl. Sci. 23 (2013) 199-214.

5. L. Beirão da Veiga, F. Brezzi and L. D. Marini, Virtual elements for linear elasticity problems, SIAM J. Numer. Anal. 51 (2013) 794-812.

6. L. Beirão da Veiga, F. Brezzi, L. D. Marini and A. Russo, The hitchhiker's guide to the virtual element method, Math. Models Methods Appl. Sci. 24 (2014) 1541-1573.

7. L. Beirão da Veiga, F. Brezzi, L. D. Marini and A. Russo, Virtual element methods for general second order elliptic problems on polygonal meshes, Math. Models Methods Appl. Sci. 26 (2016) 729-750.

8. L. Beirão da Veiga, C. Lovadina and D. Mora, A virtual element method for elastic and inelastic problems on polytope meshes, Comput. Methods Appl. Mech. Eng. 295 (2015) 327-346. 
9. L. Beirão da Veiga, C. Lovadina and A. Russo, Stability analysis for the virtual element method, arXiv:1607.05988.

10. L. Beirao da Veiga, C. Lovadina and G. Vacca, Divergence free virtual elements for the Stokes problem on polygonal meshes, ESAIM: Math. Models Numer. Anal. 51 (2017) 509-535.

11. M. F. Benedetto, S. Berrone, A. Borio, S. Pieraccini and S. Scialò, A hybrid mortar virtual element method for discrete fracture network simulations, J. Comput. Phys. 306 (2016) 148-166.

12. M. F. Benedetto, S. Berrone, S. Pieraccini and S. Scialò, The virtual element method for discrete fracture network simulations, Comput. Methods Appl. Mech. Eng. 280 (2014) 135-156.

13. S. Bertoluzza, Substructuring preconditioners for the three fields domain decomposition method, Math. Comput. 73 (2004) 659-689.

14. S. C. Brenner, Q. Guan and L.-Y. Sung, Some estimates for virtual element methods, to appear in Comput. Methods Appl. Math.

15. F. Brezzi, R. S. Falk and L. D. Marini, Basic principles of mixed virtual element methods, ESAIM: Math. Model. Numer. Anal. 48 (2014) 1227-1240.

16. F. Brezzi and L. D. Marini, Virtual element methods for plate bending problems, Comput. Methods Appl. Mech. Eng. 253 (2013) 455-462.

17. E. Caceres and G. N. Gatica, A mixed virtual element method for the pseudostressvelocity formulation of the Stokes problem, IMA J. Numer. Anal. 37 (2017) 296-331, doi:10.1093/imanum/drw002.

18. A. Cangiani, E. H. Georgoulis and P. Houston, hp-version discontinuous Galerkin methods on polygonal and polyhedral meshes, Math. Models Methods Appl. Sci. 24 (2014) 2009-2041.

19. D. Di Pietro and A. Ern, A hybrid high-order locking-free method for linear elasticity on general meshes, Comput. Methods Appl. Mech. Eng. 283 (2015) 1-21.

20. J. Droniou, R. Eymard, T. Gallouët and R. Herbin, Gradient schemes: A generic framework for the discretisation of linear, nonlinear and nonlocal elliptic and parabolic equations, Math. Models Methods Appl. Sci. 23 (2013) 2395-2432.

21. Q. Du, V. Faber and M. Gunzburger, Centroidal Voronoi tessellations: Applications and algorithms, SIAM Rev. 41 (1999) 637-676.

22. T. Dupont and R. Scott, Polynomial approximation of functions in Sobolev spaces, Math. Comput. 34 (1980) 441-463.

23. A. L. Gain, G. H. Paulino, S. D. Leonardo and I. F. M. Menezes, Topology optimization using polytopes, Comput. Methods Appl. Mech. Eng. 293 (2015) 411-430.

24. A. L. Gain, C. Talischi and G. H. Paulino, On the virtual element method for threedimensional linear elasticity problems on arbitrary polyhedral meshes, Comput. Methods Appl. Mech. Eng. 282 (2014) 132-160.

25. P. Grisvard, Singularities in Boundary Value Problems, Recherches en Mathématiques Appliquées [Research in Applied Mathematics], Vol. 22 (Springer-Verlag, 1992).

26. J.-L. Lions and E. Magenes, Problèmes Aux Limites Non Homogènes et Applications. Vol. 1, Travaux et Recherches Mathématiques, Vol. 17 (Dunod, 1968).

27. D. Mora, G. Rivera and R. Rodríguez, A virtual element method for the Steklov eigenvalue problem, Math. Models Methods Appl. Sci. 25 (2015) 1421-1445.

28. I. Perugia, P. Pietra and A. Russo, A plane wave virtual element method for the Helmholtz problem, ESAIM: M2AN 50 (2016) 783-808, doi:10.1051/m2an/2015066.

29. A. Rand, A. Gillette and C. Bajaj, Interpolation error estimates for mean value coordinates over convex polygons, Adv. Comput. Math. 39 (2013) 327-347. 
30. S. L. Sobolev, Partial Differential Equations of Mathematical Physics (Pergamon, 1964), translated from The Russian.

31. N. Sukumar and A. Tabarraei, Conforming polygonal finite elements, Int. J. Numer. Methods Eng. 61 (2004) 2045-2066.

32. C. Talischi and G. H. Paulino, Addressing integration error for polygonal finite elements through polynomial projections: A patch test connection, Math. Models Methods Appl. Sci. 24 (2014) 1701-1727.

33. G. Vacca, Virtual element methods for hyperbolic problems on polygonal meshes, Comput. Math. Appl. 74 (2017) 882-898, doi:10.1016/j.camwa.2016.04.029.

34. P. Wriggers, W. T. Rust and B. D. Reddy, A virtual element method for contact, Comput. Mech. 58 (2016) 1039-1050. 